\title{
The Deeper, Wider, Faster programme: exploring stellar flare activity with deep, fast cadenced DECam imaging via machine learning
}

\author{
S. Webb, ${ }^{1,2 \star}$ C. Flynn,,${ }^{1,2}$ J. Cooke, ${ }^{1,2,3}$ J. Zhang, ${ }^{1,2}$ A. Mahabal ${ }^{\oplus},{ }^{4}$ T. M. C. Abbott, ${ }^{5}$ R. Allen, ${ }^{1}$ \\ I. Andreoni, ${ }^{4}$ S. A. Bird, ${ }^{6,78}$ S. Goode, ${ }^{1,2}$ M. Lochner ${ }^{9,10}$ and T. Pritchard ${ }^{11}$ \\ ${ }^{1}$ Centre for Astrophysics and Supercomputing, Swinburne University of Technology, Mail Number H29, PO Box 218, 31122 Hawthorn, VIC, Australia \\ ${ }^{2}$ ARC Centre of Excellence for Gravitational Wave Discovery (OzGrav), Hawthorn, 3122, Australia \\ ${ }^{3} A R C$ Centre of Excellence for All Sky Astrophysics in 3D (Astro3D), Hawthorn, 3122, Australia \\ ${ }^{4}$ Division of Physics, Mathematics and Astronomy, California Institute of Technology, Pasadena, CA 91125, USA \\ ${ }^{5}$ NOIRLab, Mid-Scale Observatories/Cerro Tololo Inter-American Observatory, Casilla 603, La Serena, Chile \\ ${ }^{6}$ China Three Gorges University, Yichang 443002, People's Republic of China \\ ${ }^{7}$ Center for Astronomy and Space Sciences, China Three Gorges University, Yichang 443002, People's Republic of China \\ ${ }^{8}$ Key Laboratory of Optical Astronomy, National Astronomical Observatories, Chinese Academy of Sciences, 20A Datun Road, Beijing 100101, People's \\ Republic of China \\ ${ }^{9}$ African Institute of Mathematical Sciences, Muizenburg, Cape Town 7950, South Africa \\ ${ }^{10}$ South African Radio Astronomical Observatory, Observatory, Cape Town 7295, South Africa \\ ${ }^{11}$ Center for Cosmology and Particle Physics, New York University, New York, NY 10003, USA
}

Accepted 2021 June 17. Received 2021 June 15; in original form 2021 April 20

\begin{abstract}
We present our 500 pc distance-limited study of stellar flares using the Dark Energy Camera as part of the Deeper, Wider, Faster programme. The data were collected via continuous 20-s cadence $g$-band imaging and we identify 19914 sources with precise distances from Gaia DR2 within 12, $3 \mathrm{deg}^{2}$, fields over a range of Galactic latitudes. An average of $\sim 74$ min is spent on each field per visit. All light curves were accessed through a novel unsupervised machine learning techniques designed for anomaly detection. We identify 96 flare events occurring across 80 stars, the majority of which are M dwarfs. Integrated flare energies range from $\sim 10^{31}-10^{37} \mathrm{erg}$, with a proportional relationship existing between increased flare energy with increased distance from the Galactic plane, representative of stellar age leading to declining yet more energetic flare events. In agreement with previous studies we observe an increase in flaring fraction from M0 to M6 spectral types. Furthermore, we find a decrease in the flaring fraction of stars as vertical distance from the galactic plane is increased, with a steep decline present around $\sim 100 \mathrm{pc}$. We find that $\sim 70$ per cent of identified flares occur on short time-scales of $<8 \mathrm{~min}$. Finally, we present our associated flare rates, finding a volumetric rate of $2.9 \pm 0.3 \times 10^{-6}$ flares $\mathrm{pc}^{-3} \mathrm{~h}^{-1}$.
\end{abstract}

Key words: methods: data analysis - parallaxes - stars: flare-stars: low-mass.

\section{INTRODUCTION}

Stellar flares occur stochastically across main-sequence stars, commonly seen in those with large convective envelopes, and result from violent reconnection of magnetic field lines (Lacy, Moffett \& Evans 1976; Pettersen 1989; Haisch, Strong \& Rodono 1991). This sudden release of magnetic energy into the surrounding stellar atmosphere causes rapid dissipation of chromospheric and coronal field and sheet aligned currents, generating strong emission seen in the radio through to X-ray, with the largest emission occurring within the ultraviolet continuum (Lin \& Hudson 1976; Hawley \& Pettersen 1991; Osten et al. 2005; Berger et al. 2008). To date, stellar flares have been found to occur across a vast range of energies, spanning from nanoflares of $10^{23} \mathrm{erg}$ recorded from our own Sun by Parnell \& Jupp (2000), to superflares releasing upwards of $\sim 10^{33}-10^{36}$ erg as discovered by Maehara et al. (2012) within the data from The Kepler Space

^E-mail: webb.sara.a@gmail.com
Telescope (Borucki et al. 2010, Kepler hereafter). The flare frequency distribution as a function of integrated flare energy is a power law with higher energy flares being less likely (Pettersen 1989; Ishida et al. 1991; Shibayama et al. 2013; Chang, Byun \& Hartman 2015).

Bopp \& Moffett (1973) first identified two apparent populations of flares, classical, and complex, based on their temporal structure, dividing them by number of peaks present, with classical flares only exhibiting one peak and complex flares having multiple peaks (see also Moffett 1974). Classification may be limited by survey cadence, where low temporal resolution of the flare event is observed. More recently Dal \& Evren (2010) further identified sub-populations of fast and slow flares from the ratio of rise to decay time, with ratios of $<3.5$ identified as slow and $>3.5$ as fast. Work by Hawley et al. (2014) showed for M dwarfs, complex flares not only have longer durations on average but also dominate the larger energy ranges seen in their sample from Kepler. This correlation between complex events and longer decay times suggests the possibility of their only being two populations, being classical/fast and complex/slow. The historical classification into four populations may be due to past 
survey cadences and target selections. However, the fast cadenced, continual imaging required to definitively test this correlation has only been achieved by few studies to date, with majority targeting specific objects for other science goals.

Stellar flares are most commonly generated by low-mass stars. These cooler stars have larger convective envelopes surrounding their radiative cores, with theoretical models showing a star's structure transitioning into a fully convective regime at a stellar mass of $\sim 0.3 \mathrm{M}_{\odot}$ (Dorman, Nelson \& Chau 1989; Chabrier \& Baraffe 1997; Morales et al. 2009). This effect plays a part in the observed M4 transition, where stellar activity increases dramatically between M3 and M5 stellar types. Stellar activity from these stars produces flares with hot (blue) thermal components, providing a photometrically dramatic contrast against their cool red photosphere (West \& Hawley 2008; Hilton et al. 2010; Pineda et al. 2013). This dramatic contrast in thermal energy allows for easier detection, in comparison to flares of similar energy releases from hotter (bluer) stars.

Several studies into flare properties have taken advantage of $\mathrm{M}$ dwarf properties to further understand flare activity as a function of age, duty cycles, flare rate as functions of Galactic latitude, rotation period, and light-curve shape (Hawley \& Pettersen 1991; Kowalski et al. 2009; Hilton et al. 2010; Davenport et al. 2012; Maehara et al. 2012; Pineda et al. 2013; Davenport 2016; Schmidt et al. 2018; Günther et al. 2020). Notably, work by Kowalski et al. (2009) examined $~ 50000$ light curves from $10497 \mathrm{M}$ dwarfs, collected via the Sloan Digital Sky Survey (SDSS) of Stripe 82. Kowalski et al. (2009) found a strong relationship between flaring stars and those stars that have strong $\mathrm{H} \alpha$ emission during quiescence, with the large majority of flare events occurring on late-type (and younger) M dwarfs of subtype M4-M6. Kowalski et al. (2009) also confirmed that there was a trend towards higher energetic flares occurring on the relatively inactive higher mass stars, M0-M1. These results were verified by Hilton et al. (2010) using the SDSS low-mass star spectroscopic sample of $38000 \mathrm{M}$ dwarfs from West et al. (2008) to identify a further 63 flares. Duty cycles of these flares show a large increase with spectral subtype, increasing from 0.02 per cent to 3 per cent for early and late $\mathrm{M}$ dwarfs, respectively. The SDSS photometric and spectroscopic samples were also used to explore the relationship between flare rate and Galactic latitude, both finding that nearly all flares in their respective samples occurred near the Galactic plane, indicating the younger, later type $\mathrm{M}$ dwarfs, are more predominant within stellar populations of the plane (Kowalski et al. 2009; Hilton et al. 2010). Later work on SDSS DR7 M dwarf magnetic activity by Pineda et al. (2013) further confirmed that the active flare fraction decreases with greater radial distances from the Galactic centre due to stellar age.

The space-based Kepler survey has provided the largest flare catalogues to date, primarily exploring flare activity across solar-like stars during the original mission (Walkowicz et al. 2011; Hawley et al. 2014; Davenport 2016; Van Doorsselaere, Shariati \& Debosscher 2017; Yang \& Liu 2019). While the modified observing strategy implemented during K2 (the second Kepler mission created due to the failure of reaction mechanisms) allowed for the study of 134 and 540 bright $\mathrm{M}$ dwarfs by Stelzer et al. (2016) and Yang et al. (2017), respectively. More recently, with the launch of the Transiting Exoplanet Survey Satellite (Ricker et al. 2009), Günther et al. (2020) uncovered a further 673 flaring $M$ dwarfs via the 2-min cadence data. Several ground-based surveys, including the Next Generation Transit Survey (Wheatley et al. 2018), EVRYSCOPE (Law et al. 2014), and All-Sky Automated Survey for Supernovae (Shappee et al. 2012) have also produced detailed flare catalogues for bright nearby stars (Howard et al. 2018; Jackman et al. 2018; Dillon et al.
2020; Rodríguez Martínez et al. 2020a). The recent work by Chang, Wolf \& Onken (2020) investigates M dwarf flare activity across the southern sky using the SkyMapper Southern Survey DR1. Chang et al. (2020) and find 254 flare events and a steep decline in flaring fraction towards larger vertical distances from the Galactic plane.

The Deeper, Wider, Faster programme (DWF; Cooke et al., in preparation) commenced in 2014 and has targeted several fields with deep, fast 20-s cadenced optical imaging, which provides a unique opportunity to study flares over temporally resolved time frames. Here, we present the findings of a $500 \mathrm{pc}$ distance limited flare study across 12 DWF fields. Section 2 describes the DWF and optical data collection. Section 3 outlines our analysis and methodology for flare identification and the calculation of flare characteristics. The results and discussion are presented in Section 4, and conclusions are provided in Section 5.

\section{DATA}

We describe the DWF, the targeted fields, and the criteria used when choosing them in Section 2.2, and the nature of our fast cadenced, deep optical imaging in Section 2.3.

\subsection{The Deeper, Wider, Faster programme}

Several classes of fast (millisecond-to-hours duration) optical transient events have been discovered over the last few decades and the progenitors and physical mechanisms behind many of them are still relatively poorly known (e.g. supernovae shock breakouts and other rapidly evolving extragalatic events, see Garnavich et al. 2016; Perley et al. 2018; Prentice et al. 2018). What has limited our ability to detect and understand these events has been the capability to gather deep data in short, regular time intervals before, during and after the events as well as over a range of wavelengths, i.e. deep, wide-field, fastcadenced non-targeted surveys. The DWF has been designed with these challenges specifically in mind, constructing a multiwavelength and simultaneous observational programme of over 80 facilities to date. ${ }^{1}$ DWF takes a 'proactive' approach to transient astronomy, with multiwavelength observations of the target fields taken continuously over $1-3 \mathrm{~h}$ periods, typically over 6 consecutive days, acquiring data before, during, and after transient events. From our real-time processing, we are able to rapidly identify candidates and coordinate rapid and long-term follow-up observations. DWF was first created in 2014 and since its inception has had two commissioning runs and eight operational runs (see Andreoni \& Cooke 2019; Cooke et al., in preparation).

The unique design of DWF allows exploration of transients on millisecond-to-hours time-scales, providing further understanding into the classes of already observed fast transient events as well as exploring events theorized to occur on these time-scales. The optical component of DWF is able to explore a region of parameter space not yet reached by previous ground-based transient surveys, by taking continuous, high cadenced $20 \mathrm{~s}$ exposures, imaging with the wide-field sensitive Dark Energy Camera (DECam, FOV $=3 \mathrm{deg}^{2}$; Flaugher et al. 2015) on the $4 \mathrm{~m}$ Blanco telescope in Chile, or continuous $30 \mathrm{~s}$ exposures using the Hyper Suprime Cam (HSC, FOV $=1.8 \mathrm{deg}^{2}$; Furusawa et al. 2018; Kawanomoto et al. 2018; Komiyama et al. 2018; Miyazaki et al. 2018) on the $8 \mathrm{~m}$ Subaru telescope in Hawaii. Note: This work will only focus on the data gathered from DECam. Here, we present findings from our in-depth

\footnotetext{
${ }^{1}$ http://bit.do/DWF
} 
Table 1. The 12 DWF fields used in this work. Total time on field indicates the combined observations over the several dates observed.

\begin{tabular}{|c|c|c|c|c|c|c|}
\hline $\begin{array}{l}\text { Target } \\
\text { field }\end{array}$ & RA & Dec. & $\begin{array}{l}\text { Galactic } \\
\text { latitude }\end{array}$ & $\begin{array}{l}\text { Total time } \\
\text { on field }(\mathrm{h})\end{array}$ & $\begin{array}{l}\text { Dates } \\
\text { observed }\end{array}$ & $\begin{array}{r}\text { \# Sources }^{a} \\
(\leq 500 \mathrm{pc})\end{array}$ \\
\hline Antlia & $10: 30: 00.0$ & $-35: 20: 00.0$ & 19.172 & 7.02 & 3, 5-7 Feb 2017 & 1743 \\
\hline Dusty 10 & 10:12:00.0 & $-80: 50: 00.0$ & -19.957 & 3.50 & 7, 9 June 2018 & 1911 \\
\hline Dusty 12 & $11: 46: 00.0$ & $-84: 33: 00.0$ & -21.889 & 4.39 & 26-30 July 2016 & 2091 \\
\hline FRB131104 & $06: 44: 00.0$ & $-51: 16: 00.0$ & -21.930 & 6.37 & 14-17 Jan 2015, 18-22 Dec 2015 & 1776 \\
\hline $8 \mathrm{~h}$ & 08:16:00.0 & $-78: 45: 00.0$ & -22.618 & 4.95 & 6-9 June 2018 & 1805 \\
\hline Dusty11 & $11: 20: 00.0$ & $-85: 20: 00.0$ & -22.814 & 2.35 & 7-9 June 2018 & 1790 \\
\hline NGC 6744 & 19:08:00.0 & $-64: 30: 00.0$ & -26.054 & 18.38 & 26, 28-30 July 2016, 2-7 July 2016 & 1966 \\
\hline Prime & 05:55:07.0 & $-61: 21: 00.0$ & -30.262 & 6.88 & 14-17 Jan 2015, 2-7 Feb 2017 & 1459 \\
\hline NSF2 & $21: 28: 00.0$ & $-66: 48: 00.0$ & -39.823 & 3.64 & 26-27 July 2016 & 1453 \\
\hline FRB010724 & 01:18:06.0 & $-75: 12: 19.0$ & -41.804 & 2.63 & 18-22 Dec 2015 & 1436 \\
\hline $4 \mathrm{~h}$ & 04:10:00.0 & $-55: 00: 00.0$ & -44.756 & 8.05 & 15-17 Jan 2015, 18-22 Dec 2015 & 1252 \\
\hline $3 \mathrm{~h}$ & 03:00:00.0 & $-55: 25: 00.0$ & -53.432 & 6.88 & 18-22 Dec 2015 & 1242 \\
\hline
\end{tabular}

analysis of stellar flares discovered in the post-run archival DECam data processing.

\subsection{DWF fields used}

In this work, we use a total of 12 DWF fields, each visited multiple times between the years 2015-2018, as shown in Table 1. The total time visited on each field varied, averaging $6.30 \mathrm{~h}$ collected over several nights. A total of $75.08 \mathrm{~h}$ of observations are analysed in this work.

The ongoing DWF has targeted over 20 distinct fields, with several having repeat visits. Due to the nature of DWF, targeted fields need to have simultaneous visibility by Chilean facilities (DECam, Gemini, VLT, etc.), as well as Australian or South African radio telescopes (ASKAP, Parkes, Molonglo, MWA, MeerKAT), Antarctic telescopes (South Pole Telescope, AST3-2), and space-based facilities, as well as the growing number of other simultaneous and follow-up facilities (more detail can be found in Cooke et al., in preparation). The fields are selected using several criteria, mainly, (1) sky position, enabling common visibility for the multiwavelength telescopes to simultaneously observe during runs scheduled at different times of the year, (2) preference towards low-redshift galaxy clusters/groupings or globular clusters, (3) legacy fields with multiwavelength preimaging, and (4) fields with previously detected fast radio bursts (FRBs) and FRB repeaters, as well as the need for regions of low Galactic extinction for certain wavelength facilities. As a result, the fields targeted are somewhat arbitrarily distributed across the sky, providing data across a variety of Galactic declinations.

\subsection{DECam fast-cadence imaging}

The imaging data were collected over multiple DWF runs using DECam. During a DWF run, continuous 20-s g-band exposures are taken across multiple target fields. The use of the $g$-band filter maximizes the depth of our imaging, reaching $\sim 0.5$ mag deeper in comparison to the other filters in dark time. The expected limiting magnitude in $g$ band is $m(\mathrm{AB}) \sim 23$, for an average seeing of 1.0 arcsec and airmass of 1.5 (relatively high airmass due to the field constraints of observing simultaneously with multiple facilities). All images used in this work were post-processed through the NOAO High-Performance Pipeline System (Scott et al. 2007; Swaters \& Valdes 2007; Valdes \& Swaters 2007), and transferred to the OzSTAR supercomputer at Swinburne University of Technology.
We processed each of the $62 \mathrm{CCDs}$ in parallel, performing source extraction via SEXTRACTOR (Bertin \& Arnouts 1996), zero-point corrections and magnitude offset corrections against the SkyMapper DR2 catalogue (Bertin \& Arnouts 2010; Onken et al. 2019). A master list was compiled by cross-matching the position of all extracted sources from each CCD, over all exposures within an 0.5 arcsec radius between source centroids, producing one catalogue of unique source positions per field. We used this master catalogue for reference when selecting our volume-limited sample, as explained in detail in the following section.

\subsection{Volume-limited star selection}

In this paper, we explore flare activity out to $500 \mathrm{pc}$ across each of our 12 fields covering a total sky area of $\sim 36 \mathrm{deg}^{2}$ and volume of $\sim 443000 \mathrm{pc}^{3}$. Distances to the stars were provided by the Gaia DR2 parallaxes to pre-select our sample (Evans et al. 2018; Luri et al. 2018). For each field, we queried Gaia DR2 using Vizier, ${ }^{2}$ selecting only sources that meet the following criteria:

(i) $1000 /$ parallax (mas) $\leq 500 \mathrm{pc}$

(ii) $\Delta$ parallax/parallax (mas) $\leq 0.2$

(iii) $\mathrm{g}$ magnitude $\geq 11$

It should be noted that due to the systematic implicit biases associated with measuring parallaxes our sample of stars are more likely to be on the higher end of their associated distance \pm error (as described by Trumpler \& Weaver 1953). We choose to apply a magnitude limit cut to avoid sources likely to saturate from our DECam observations. Each Gaia source catalogue was crossmatched against each field's master source catalog, with light-curve files generated for each source existing in our DWF data. Finally, only light curves with three or more consecutive detections during a single night's visit were evaluated for flares (possibly excluding flares shorter than $40 \mathrm{~s}$ ). Table 1 outlines each of our target fields, and the number of Gaia selected sources. A total of 19914 sources were assessed. Separate light curves were generated for each source over each night of observations. The total number of light curves in the sample is 114958 .

\footnotetext{
${ }^{2}$ GAIA DR2 Vizier
} 


\section{ANALYSIS}

\subsection{Flare identification}

\subsubsection{Anomaly ranking visual inspection - candidate flagging}

To identify flares within our sample, we first use unsupervised machine leaning to rank light curves from most to least anomalous, as detailed in full in Webb et al. (2020). A set of 25 features was extracted for each light curve in our sample. The features and light curves were then fed into the PYTHON-based Astronomaly package (Lochner \& Bassett 2020). Astronomaly consists of a PYTHON back end and JavaScript front end to easily explore the data via a locally hosted web interface. We pass the light-curve features through the scikit-learn implementation of isolation forest within Astronomaly to determine the most anomalous sources (Ting, Liu \& Zhou 2008). We visually inspect all light curves through the Astronomaly GUI, shown in order of anomaly ranking (as measured by the isolation forest algorithm). All light curves with potential flares are flagged for further evaluation. We reduce the original 114958 light curves to just $\sim 700$ during this process by removing static stars using HDBSCAN clustering (for details, see Webb et al. 2020).

\subsubsection{Flare detection and criteria}

The flagged light curves were then passed through a change point analysis (CPA) algorithm to flag the start and finish positions of individual flares within the light curve. We use the method as first outlined by Chang, Byun \& Hartman (2015) to develop a flexible CPA algorithm, modified to suit our fast cadenced data. CPA is useful in identifying abrupt variations in sequential data and is done so by combining a cumulative sum scheme and bootstrap rank statistics to flag points of systematic change within sequential data. Our adaption of Chang et al. (2015) FINDflare algorithm is used to flag all possible flares within our shortlisted light curves. The criteria used within the FINDflare algorithm are as follows, N1: The required number of standard deviations above the median for the data points, N2: The number of standard deviation plus uncertainty above the median, N3: The number of consecutive points required to meet the above criteria to be flagged as a flare. We choose to use the values $\mathrm{N} 1=3, \mathrm{~N} 2=2$, $\mathrm{N} 3=3$, after sensitivity testing on known DWF flares identified from previous work by Andreoni et al. (2020) and Webb et al. (2020). We then estimate the quiescent magnitude of each source by taking the median magnitude of all data points before the flare (and in very rare cases of flares at the beginning of the observations, after the flare). We then determine the standard deviation, $\sigma$, of the quiescent section of the light curve, and as set with the N3 value, require at least three consecutive data points to be greater than the two standard deviations above the median magnitude, see Fig. 1 for example. All light curves that passed these criteria were manually inspected alongside their image cut-outs for each exposure. This stage of careful image evaluation allowed the rejection of candidates which were caused due to observational effects (e.g. cosmic rays, hot pixels, edge detections).

\subsection{Flare characteristics}

For each flare in our sample, we record several characteristics of the event. These include, duration, maximum change in magnitude $(\Delta M)$, flare type, and equivalent duration.

The duration of the flare is measured from the last observed quiescent point. The first data point, of two consecutive data points, to
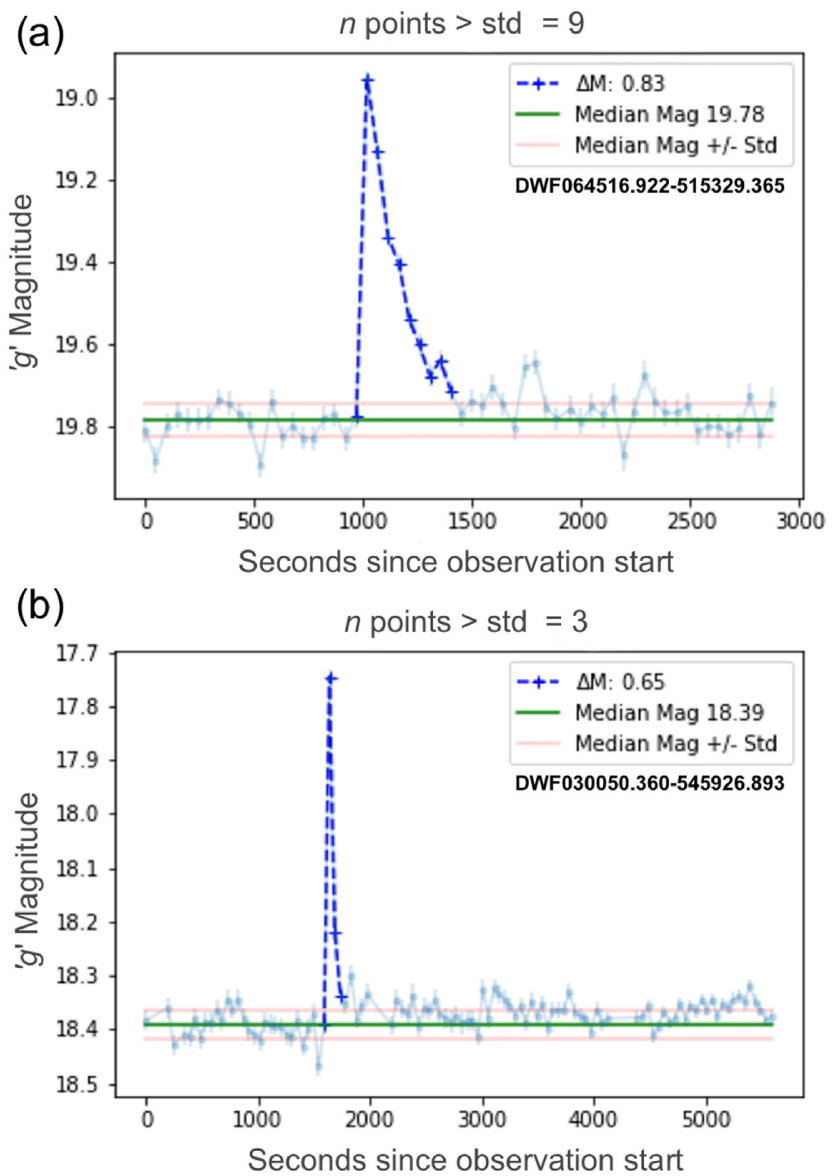

Figure 1. Classical and short-duration flare examples. (a) An example flare with nine consecutive data points greater than one standard deviation (red line) above the median magnitude (green line) of the quiescent source. (b) An example short-duration flare with three consecutive data points greater than one standard deviation (red line) above the median magnitude (green line) of the quiescent source.

fall below the upper $1 \sigma$ uncertainty to the mean quiescent magnitude, two examples can be seen in panel (a) of Fig. 2. In the rare event of our observations finishing before the flare reaches the star's quiescent magnitude, the duration is a minimum limit and marked accordingly in Table A1 and an example can be seen in panel (b) of Fig. 2.

The $\Delta M$ of each flare is calculated by the difference between the peak magnitude recorded and the median quiescent magnitude for each source. Here it is important to note that these measurements can underestimate the true value of $\Delta M$ which could occur during the 20-30 s readout after each exposure and diluted by the $20 \mathrm{~s}$ exposure itself. This is more likely for the short duration flares. Fig. 1 demonstrates both longer duration (a) and short duration flares (b). These characteristics are recorded further in Table A1.

\subsection{Integrated flare energy}

The integrated flare energy represents the total amount of energy released over the duration of the flare. To calculate the total integrated flare energy, we first calculate the $g$-band component from our observations.

To estimate total energy in $g$ band, each light curve was normalized by setting the star's quiescent flux to one, creating a relative output for each data point. Using the relative outputs over the duration 

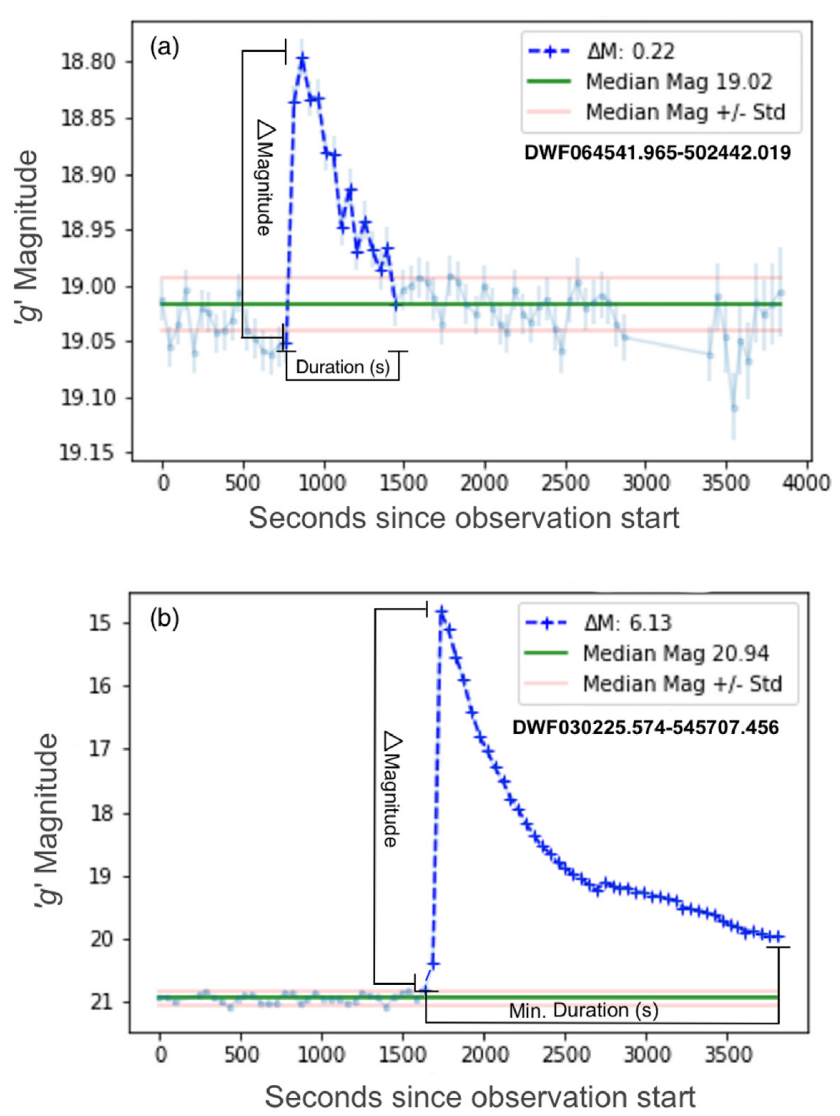

Figure 2. (a) Flare star example illustrating the maximum magnitude change and total event duration definitions. The maximum change in magnitude is determined by the difference between the peak flare detection and the median quiescent magnitude (green line; see definition of the quiescent mag described in Section 3.1.2). The total duration of the event is measured from the last detection of a quiescence magnitude before the flare, to the point at which the flare re-enters within $1 \sigma$ of the mean quiescent magnitude range (red lines). (b) A flare event example with the flare continuing past the observational period, permitting only a minimum duration to be recorded.

of the flare, we sum the total excess amount of flux generated by the flare event, $\sum_{\text {rel }}$. Fig. 3 demonstrates a flare's excess flux for each point (relative intensity $>1$ ). We use the Riemann sum binning method across all points. For each flare we first derived the relative intensity for each point in the light curve from the magnitudes with the following:

$$
\frac{I_{\mathrm{o}+\mathrm{f}}}{I_{\mathrm{o}}}=10^{\frac{\Delta \mathrm{mag}}{2.5}},
$$

where $I_{\mathrm{o}+\mathrm{f}}$ and $I_{\mathrm{o}}$ are the intensity values of the flaring and the quiescent stellar surfaces in the observed $g$ band. Note, this method may underestimate the true local flux by a small amount small spikes in flux after reaching the quiescent level threshold are not included. For each detection of the flare, the relative intensity is integrated over the flare duration to calculate the equivalent duration:

$\varepsilon_{\mathrm{f}}=\int_{t_{1}}^{t_{2}}\left(\frac{I_{\mathrm{o}+\mathrm{f}}(t)}{I_{\mathrm{o}}}-1\right) \mathrm{d} t$.

The quiescent stellar fluxes for $g$ band, $F_{\lambda}$, are computed taking the median quiescent magnitude of the source, and computing the flux

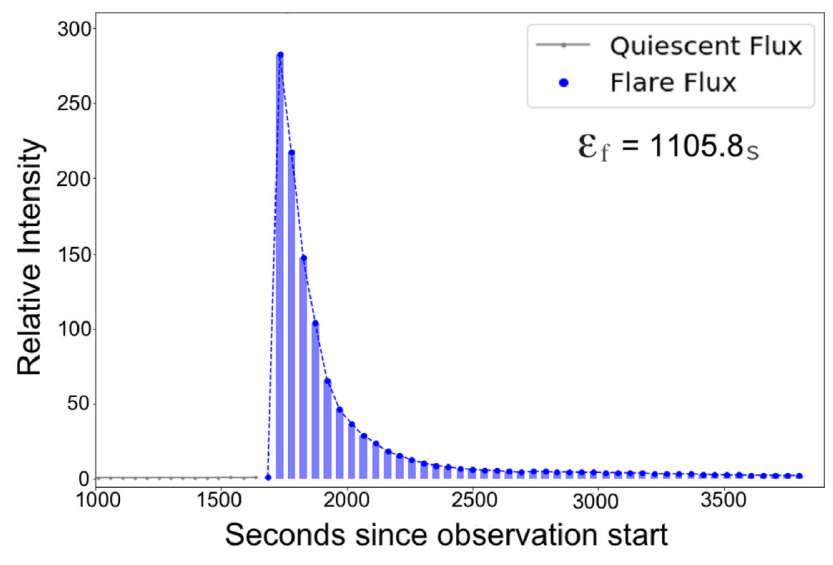

Figure 3. An example of normalization and calculation of equivalent duration for flare events. Displayed is the normalized light curve for DWF030225.574-545707.456. The total equivalent duration is the sum of the detections. The total equivalent duration of this flare is $\varepsilon_{\mathrm{f}}=1105.8 \mathrm{~s}$.

$\left(\mathrm{W} \mathrm{m}^{-2}\right.$ ) for the specific wavelength of $430 \mathrm{~nm}$ via the open-source Gemini Observatory conversion tool. ${ }^{3}$

The flux at source is then calculated with the following:

$F_{*}=\left(1000 \times F_{\lambda}\right) \times\left(4 \pi d^{2}\right)$,

where $d$ is distance to star. Finally, the total integrated $g$-band flare energy is calculated by multiplying the relative flare energy by the quiescent stellar flux and the duration of the flare:

$E_{\mathrm{f}(g \text {-band })}=\varepsilon_{\mathrm{f}} F_{*} t$,

where $t$ is duration of the flare in seconds.

To estimate the total integrated flux across all wavelengths of the flare, we modelled the expected blackbody emission for a typical flare. Flare temperatures have been found to vary dramatically across similar spectral types and energy ranges. Commonly, the combined line and continuum emission of flares has been approximated by a $\sim 9000 \mathrm{~K}$ blackbody (Osten \& Wolk 2015). However, recent work by Howard et al. (2020) found that across 42 K5-K5 superflares, 43 per cent of flares emit temperatures above $14000 \mathrm{~K}$. In this work will assume a likely flare temperature of $9000 \mathrm{~K}$.

To calculate the overall contribution of the $g$-band flux emission, we produce a Planck function for $T=9000 \mathrm{~K}$ and compute the ratio of radiance over the DECam $g$-band width (400-560 nm), taking into account the relative transmission across the filter, and compare it to the full emission, see Fig. 4. From this we find that the total emission across our $g$ band accounts for 10.9 per cent of the total energy released. We therefore multiply the $E_{\mathrm{f}(g \text { band })}$ by 9.2 to account for the full bolometric energy $\left(E_{\mathrm{f}(\mathrm{bol})}\right)$.

\section{RESULTS AND DISCUSSION}

In our sample of 19914 stars, we identified a total of 96 flares, from 80 individual stars, within our DWF 500 pc distance limited sample. We breakdown our flare results across source spectral type, Galactic latitude, stellar age, and flare duration. We further explore the flare frequency distribution (FFD) of our sample, and present our flare rate calculations.

\footnotetext{
${ }^{3} \mathrm{https} / / /$ www.gemini.edu/sciops/instruments/midir-resources/imaging-cali
} brations/fluxmagnitude-conversion 


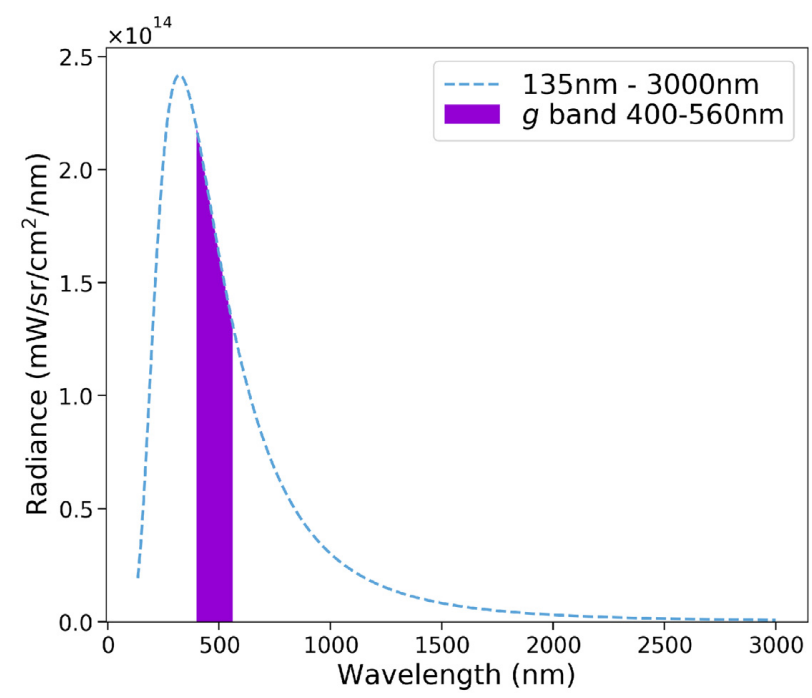

Figure 4. Planck function of spectral radiance of a $9000 \mathrm{~K}$ object. The $g$ band emission component $(400-560 \mathrm{~nm})$ is highlighted purple. Note that not all emission from across the $400-560 \mathrm{~nm}$ is included in the ratio calculation, as it was corrected for relative transmission of flux in $50 \mathrm{~nm}$ blocks from the DECam $g$ filter. Function generated using RADIS PYTHON package.

\subsection{Spectral types}

Using Gaia g magnitudes and BP - RP colour information, we identified the likely spectral type for each source in our sample, identifying that this work primarily explores G-M6 spectral types.
Flaring sources were identified across the K to M6 range, with the majority occurring across the M2-M5 range (Fig. 5). Fig. 6(a) shows the number of stars as a function of spectral type in our $500 \mathrm{pc}$ sample and those which flared, and (b) presents the flaring fraction for each of the binned spectral types. We find an increase in flaring fraction across the $\mathrm{M}$ dwarfs, with it peaking in our sample at $\sim 14$ per cent for M6. Although the overall flaring fractions are moderately lower than previous work ( $\sim 30$ per cent lower for M5), we still observe a steep increase from M4 onward, coinciding with where it is thought M dwarfs become fully convective (Kowalski et al. 2009; Yang et al. 2017; Günther et al. 2020; Mondrik et al. 2019; Rodríguez Martínez et al. 2020b).

Our lower flaring fractions may be due to limitations on continuous time on target fields, with an average of $\sim 74$ min on field per night. We speculate that we are unable to observe the full range of classical longer duration flares (e.g. Hawley et al. 2014; Günther et al. 2020), through our observation strategy and selection criteria (e.g. the flare peak must be within the data). As discussed in more detail in Section 4.5, our flare sample predominately contains shorter duration events.

Using the likely spectral types from Fig. 5, we investigated the colour-colour temperature relationship previously presented in (Schmidt et al. 2016). We use the All WISE (Cutri et al. 2021) $W 1$ (3.35 um) and W2 (4.6 um) bands to calculate (W1 - W2). To calculate $(r-z)$ we used the SkyMapper DR3 (Wolf et al. 2018; Onken et al. 2019) and $z$-band observations for each of our flaring sources. Both $(W 1-W 2)$ and $(r-z)$ values for sources can be found in Appendix A. The resulting colour-colour plot can be seen in Fig. 7. We use the BP - RP colour temperature relationship as outlined by Casagrande et al. (2020) to estimate the effective temperature $\left(T_{\text {eff }}\right)$

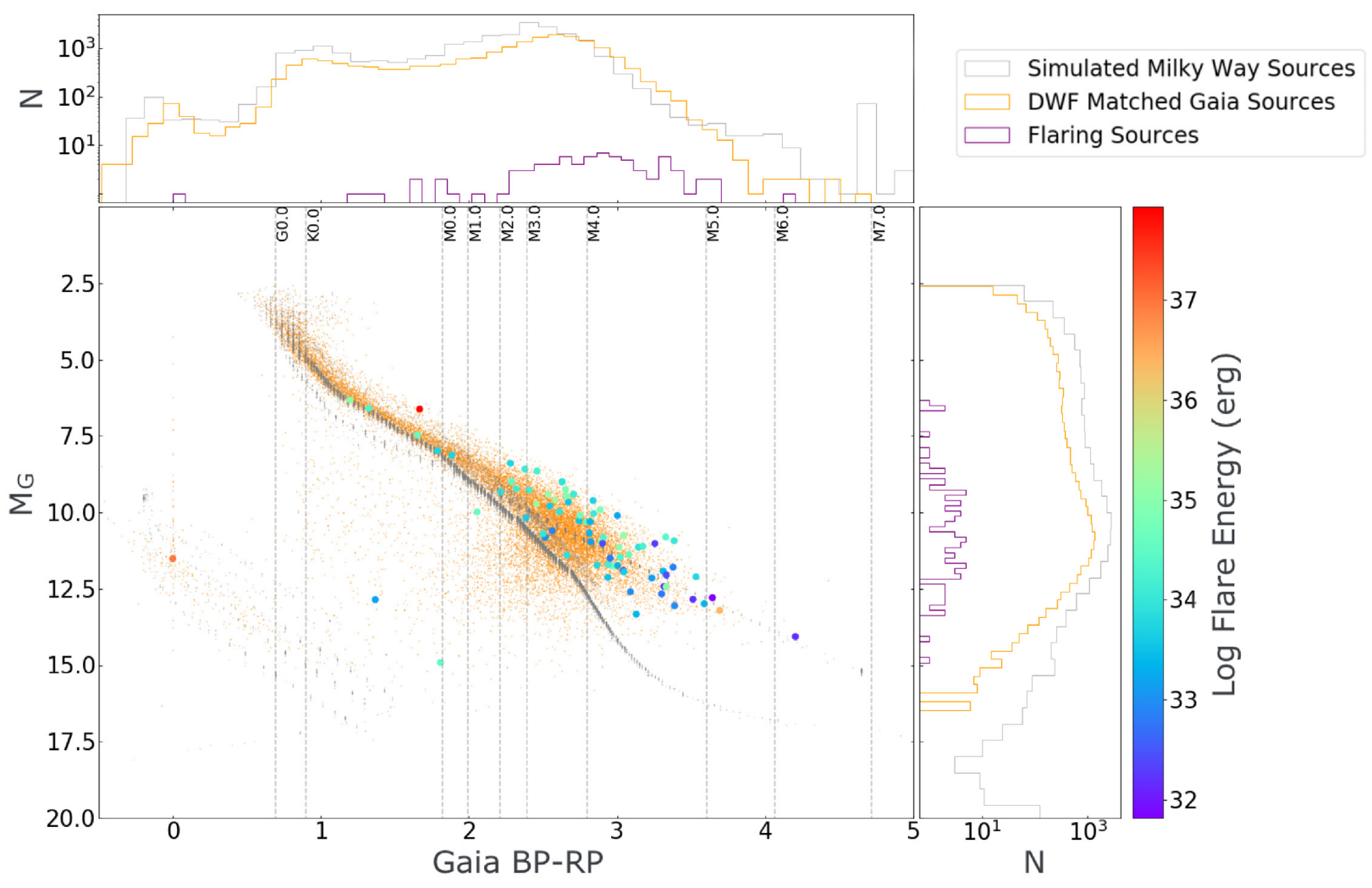

Figure 5. Colour-magnitude diagram representing all sources within this study (orange $\odot$ ), simulated sources for this study's fields (black + ), and the identified flaring sources $(\bullet)$. The simulated sources were retrieved from Vizier's online catalogue of the Milky Way stars from the GUMS. The histograms for both the $x$ and $y$-axis show in more detail the distributions of sources across our sample, simulated sample and specifically flares (Robin et al. 2012). 

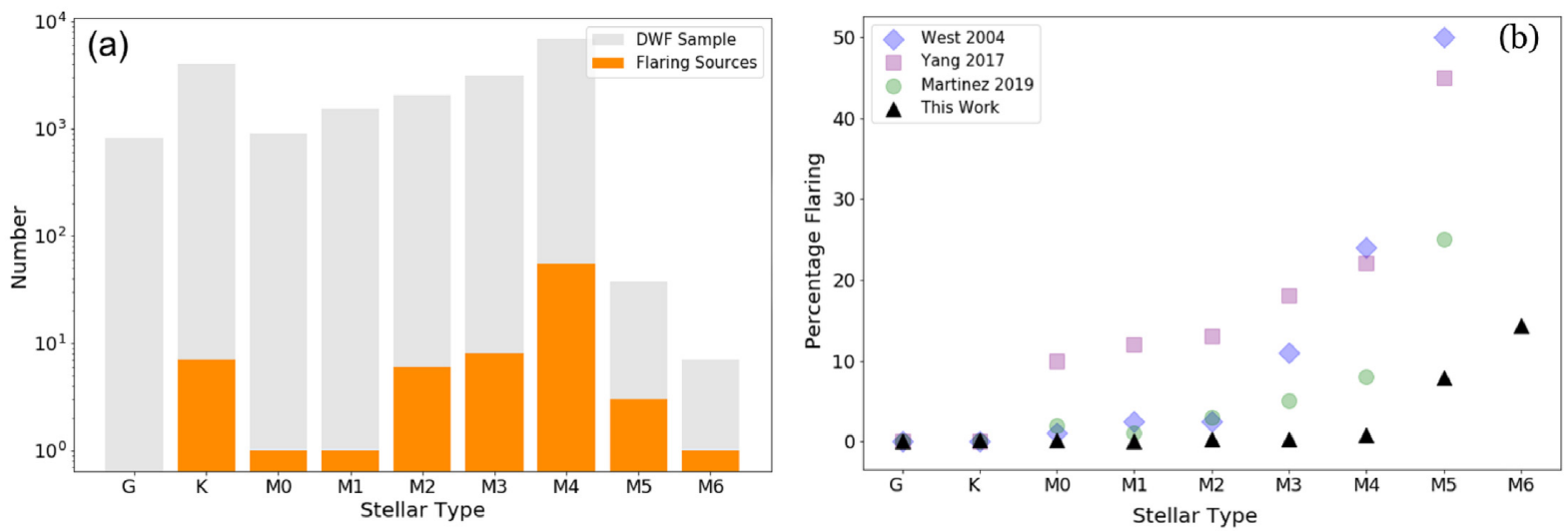

Figure 6. (a) Spectral type distribution for full DWF sample and the flaring sources. The spectral types are derived from the intrinsic colour in $V-I$ from Ducati et al. (2001) by applying the transformation GBP - GRP $=(V-I)+0.15$, as outlined in Mould, Clementini \& Da Costa (2019). (b) Percentage of flaring sources versus spectral type, with the spectral type determined in the same manner as the previous panel. Values as identified by West et al. (2004), Yang et al. (2017), and Rodríguez Martínez et al. (2020b) are also plotted as diamonds, squares, and circles, respectively.

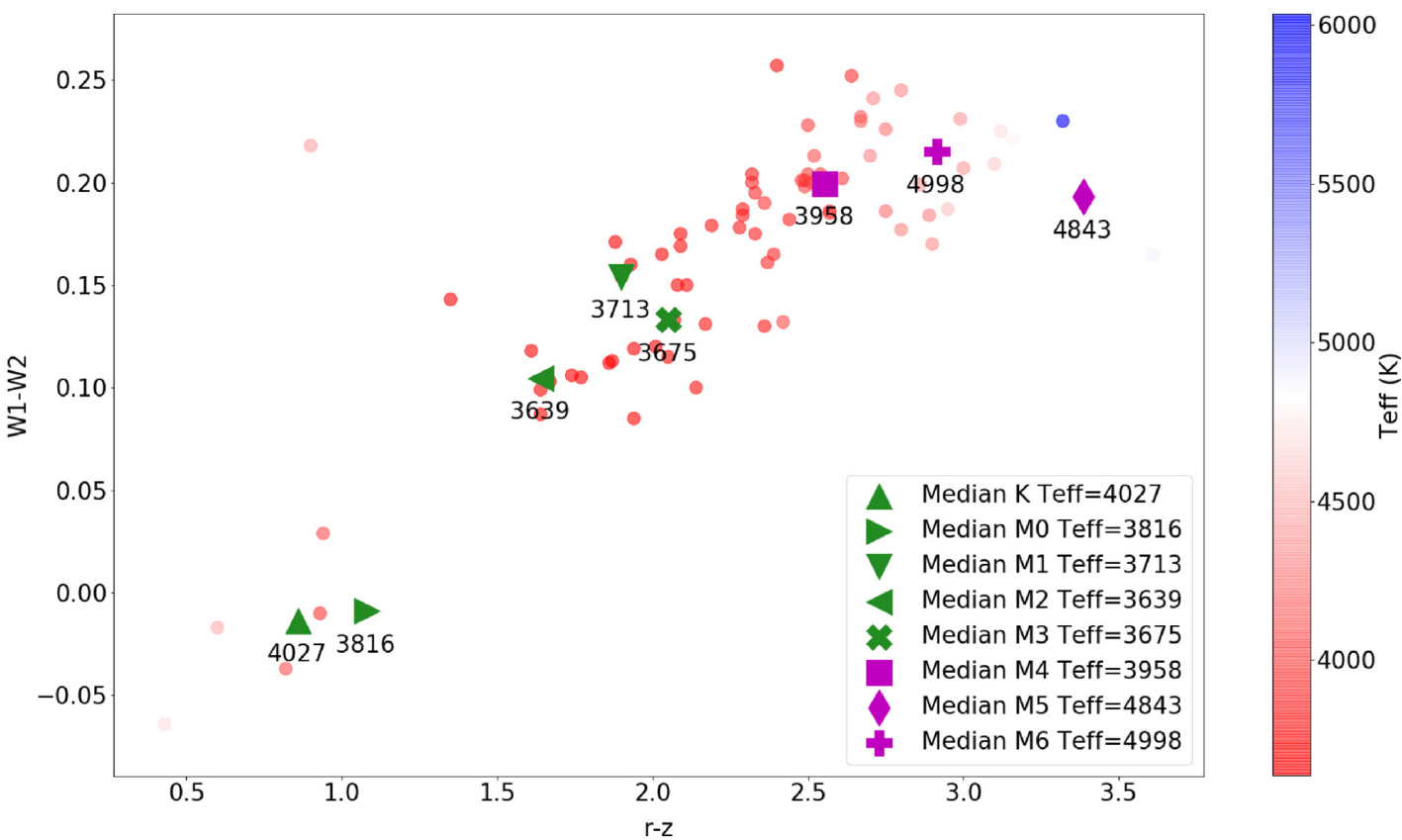

Figure 7. Colour-colour diagram of $(W 1-W 2)$ versus $(r-z)$ for DWF flares plotted with circles mapped to $T_{\text {eff. }} T_{\text {eff }}$ was derived for each source using the Gaia (BP - RP) colour to temperature relationship outlined in Casagrande et al. (2020). The median positions for each spectral class is plotted with green markers. The colour-temperature relationship breaks down for sources with (BP $-\mathrm{RP})>2.5$ and those spectral classes effected have their median position markers coloured in red. The temperature relationship presented by Schmidt et al. (2016) is shown with black lines and labelled.

for each of our flaring sources, coloured accordingly in Fig. 7. For each spectral type, we calculate the median $(\mathrm{W} 1-\mathrm{W} 2)$ and $(r-z)$ positions and $T_{\text {eff }}$, plotted in green markers. The estimates for $T_{\text {eff }}$ breakdown for redder sources and are not reliable for sources with $(B P-R P)>2.5$. Those spectral types which have untrustworthy temperature estimates are presented by red markers. We expand the initial temperature relationship presented by (Schmidt et al. 2016), probing to the M3 range, however are unable to present reliable temperature colour relationships for M4-M6 range.

\subsection{Flare energies}

Fig. 8 shows flare duration versus integrated flare energy for our sample of flares alongside additional flare samples from various surveys. We identify a population of low-energy flares, bridging the gap between $X$ class solar flares $\left(\sim 10^{31} \mathrm{erg}\right)$ and the majority of stellar flares as catalogued by Kepler and Galaxy Evolution Explorer $(G A L E X)\left(\sim 10^{35} \mathrm{erg}\right)$. These low-energy flares have a small range in duration, occurring over $\sim 5-30$ min periods. Interpreting the scaling relations in Fig. 8 indicates our sample is especially diverse, spanning magnetic field strengths of 60 to several hundred Gauss and active region length-scales of $10^{9} \mathrm{~cm}$ to exceeding the radius of the Sun.

Interestingly, Fig. 8 also demonstrates a clear relationship between distance above the Galactic plane $Z$ and total flare energy. Our sample appears to show flares further from the Galactic plane have larger total integrated flare energy. Total flare energy also tends to be larger for longer duration flares. 


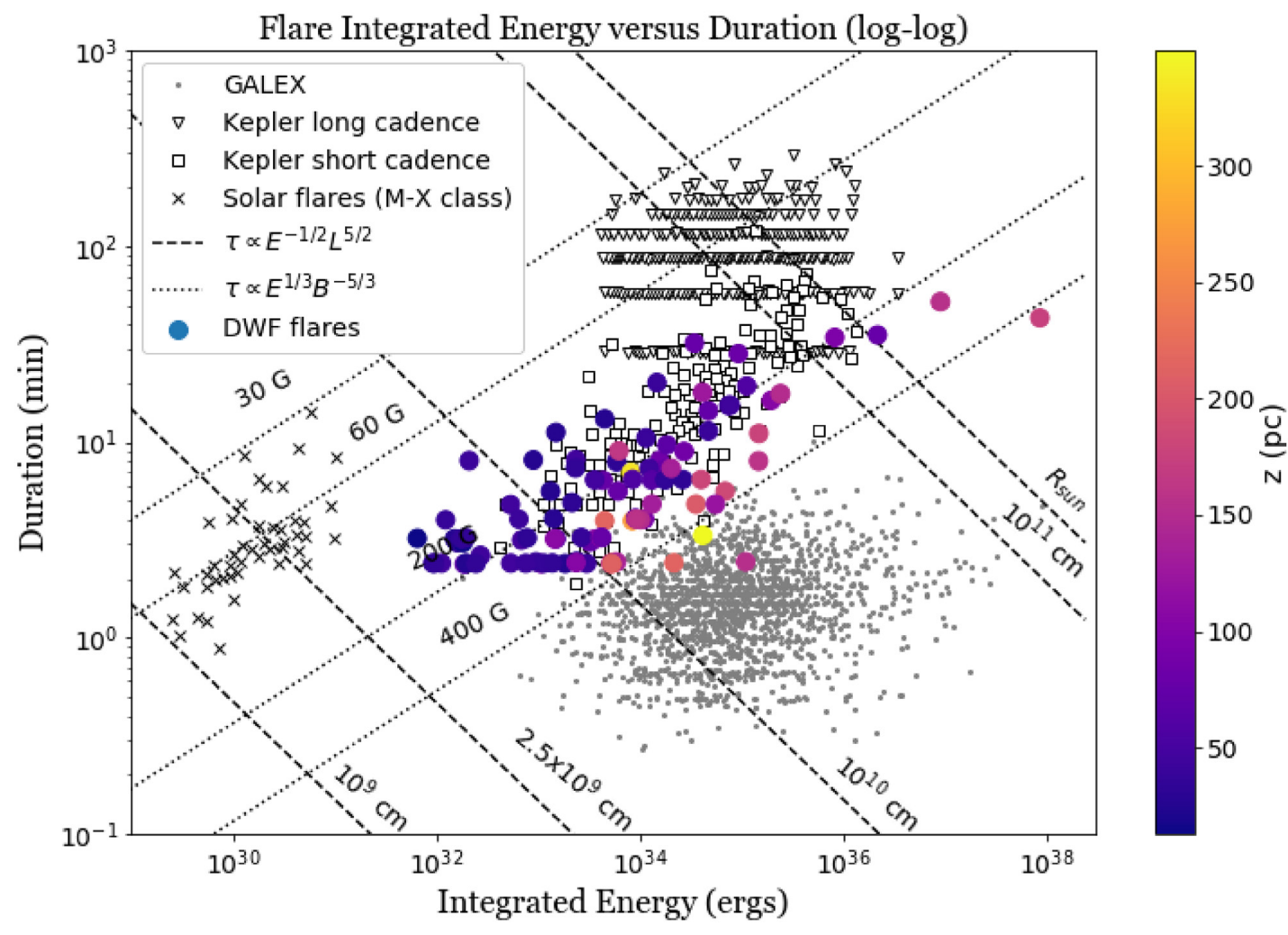

Figure 8. Duration versus flare energy for the DWF sample $(\bullet)$ with colour representing absolute distance $|Z|$ from Galactic plane. Additionally plotted are white light solar and stellar flare datasets from Namekata et al. (2017) (×), Kepler superflares flares identified in long cadence data by Shibayama et al. (2013) $(\nabla)$, Kepler superflares identified in short cadence data by Maehara et al. (2015) ( $\square$ ), and GALEX short duration flares identified by Brasseur, Osten \& Fleming (2019) (.). The dotted and dashed lines show the theoretical scaling laws derived in Namekata et al. (2017), where $B$ is magnetic field strength in the flaring region, and $L$ is the flare length scale.

We find that the distribution of flare energies is bi-modal as shown in Fig. 9, with this grouping of lower energy flares being centralized around sources $\lesssim 100 \mathrm{pc}$ from the Galactic plane. To ensure that this result was not due to observational selection effects, we took the detected flares with distances $<100 \mathrm{pc}$ and simulated them at increasing distances out to $500 \mathrm{pc}$. To do this, we incrementally added $100 \mathrm{pc}$ to the source distances, creating four samples in the following ranges, 100-200, 200-300, 300-400, 400-500 pc. To calculate the magnitudes of each source at each simulated distance, we used the following:

$m=5 \times \log _{10}(d+x / d)+q_{\mathrm{mag}}$,

where $m$ is the magnitude at the simulated distance, $d$ is the original distance of the flaring source, $x$ is the additional distance added (e.g. $100 \mathrm{pc}, 200 \mathrm{pc}$ etc.), and $q_{\mathrm{mag}}$ is the original quiescent magnitude of the flaring source. The difference between $m$ and $q_{\mathrm{mag}}$ is calculated and subsequently applied for each point in the original light curve and newly associated magnitude errors were calculated. The magnitude errors were modelled using a DECam magnitude error versus magnitude plot (for a typical night of average seeing and airmass, see Fig. B1). The simulated sample was put through the identical flare identification pipeline, described in Section 3.1.

We find 95 per cent of our $<100$ pc flare sample were recovered, when simulated between 100 and 200 pc distances. The recovery rate continues to drop out to $500 \mathrm{pc}$, reaching 60 per cent, see Fig. 10. If we were assume the stellar population is isotropic in our sample, we would expect to see a $\sim 30-40$ per cent more flares at these distances then our study observes, suggesting a drop off of low-energy flares due to the young disc. Via these tests we also confirm that several

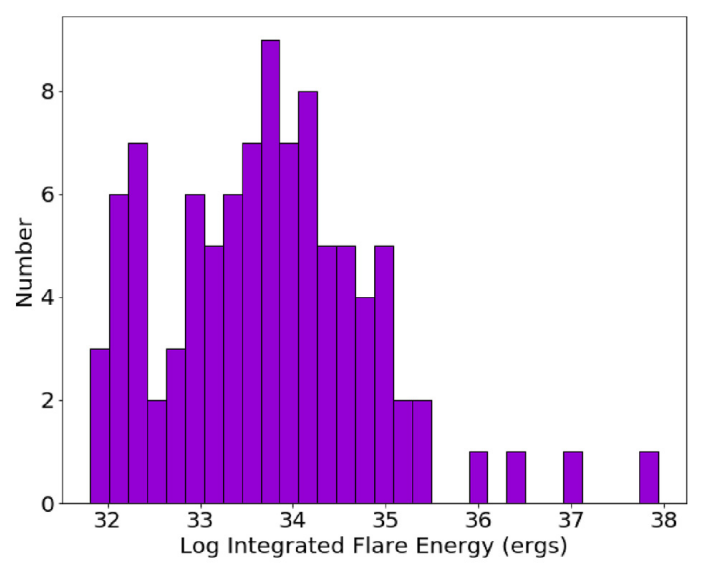

Figure 9. Distribution of $\log$ integrated flare energies, ranging from $\sim 10^{31-37} \mathrm{erg}$.

lower energy flares, $\sim 10^{31} \mathrm{erg}$ were detectable at $500 \mathrm{pc}$. However, the drop in number of low-energy flares past $\sim 100 \mathrm{pc}$ in our sample suggests an astrophysical cause, likely stellar age, further discussed in Section 4.3.

\subsection{Flares across Galactic declination}

We postulate that the results discussed Section 4.2, of densely populated flares within $\sim 100 \mathrm{pc}$, is representative of the dense population of stars within the thin disc of the Milky Way. The thin 


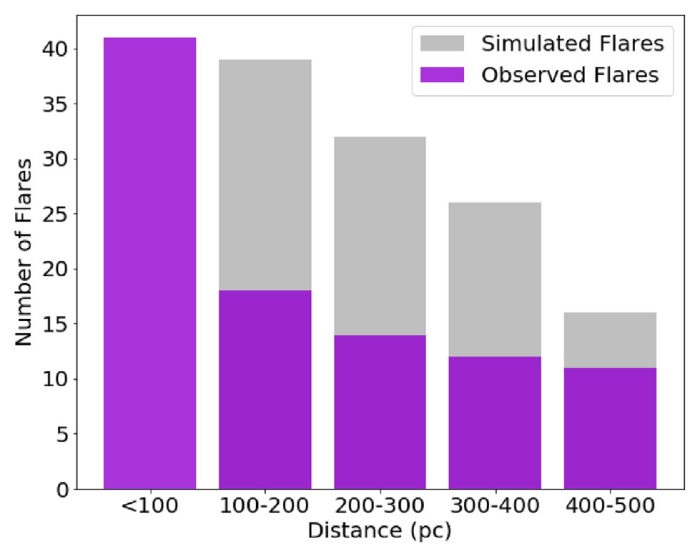

Figure 10. Number of real flares (purple) detected in our sample grouped in $100 \mathrm{pc}$ bins. The 41 flares within $0-100 \mathrm{pc}$ were simulated in $100 \mathrm{pc}$ bins out to $500 \mathrm{pc}$ and those recovered in the flare identification pipeline are represented in grey.

Table 2. DWF fields galactic latitudes, maximum distance above the Galactic plane $(|Z|)$ probed via our $500 \mathrm{pc}$ distance sample, and the average $|Z|$ of flares observed in that field.

\begin{tabular}{lcccc}
\hline Field & $\begin{array}{c}\text { Galactic } \\
\text { latitude }\end{array}$ & $\begin{array}{c}\text { Max }|Z| \text { at } \\
\text { 50 distance cut }\end{array}$ & \# of flares & $\begin{array}{c}\text { Average }|Z| \\
\text { of flares }\end{array}$ \\
\hline Antlia & 19.172 & 164.12 & 8 & 73.76 \\
Dusty10 & -19.957 & 170.57 & 4 & 52.47 \\
Dusty12 & -21.889 & 186.31 & 3 & 72.73 \\
FRB131104 & -21.93 & 186.64 & 13 & 65.50 \\
8 h & -22.618 & 192.2 & 6 & 121.15 \\
Dusty11 & -22.814 & 193.77 & 1 & 63.73 \\
ngc6744 & -26.054 & 219.50 & 27 & 81.30 \\
Prime & -30.262 & 251.86 & 16 & 111.67 \\
NSF2 & -39.823 & 320.07 & 3 & 194.78 \\
FRB010724 & -41.804 & 333.14 & 6 & 36.93 \\
4 h & -44.756 & 351.9 & 10 & 109.96 \\
3 h & -53.432 & 401.43 & 9 & 92.96 \\
\hline
\end{tabular}

disc has an estimated scale height of 200-300 pc (Cabrera-Lavers et al. 2007; Jurić et al. 2008; Abazajian et al. 2009) with the youngest stars concentrated closest to the plane (Binney \& Tremaine 1987). More specifically it is the younger $\mathrm{M}$ dwarfs that are predominant within the stellar population of the thin disc (Kowalski et al. 2009; Hilton et al. 2010; Pineda et al. 2013).

To investigate the flaring fraction as a function of the vertical distance from the Galactic plane $Z$, we use the distance, as calculated using the Gaia DR2 parallaxes (Luri et al. 2018), and each field's central Galactic latitude to determine the vertical distance from the Galactic plane. Across our 12 fields, we probe an average distance of $\sim 247$ pc off the Galactic plane. Table 2 displays the maximum $Z$ for each field, and the average $Z$ for flares identified in each field. On average the majority of flares are occurring at $\sim 1 / 3$ of the maximum $Z$ probed.

When combining data across all fields, we find a strong relationship between flaring fraction and $Z$ (normalized over the total amount of observing hours), shown in Fig. 11. This result is in good agreement with previous studies (e.g. Kowalski et al. 2009; Brasseur et al. 2019; Chang et al. 2020), that also find this relationship.

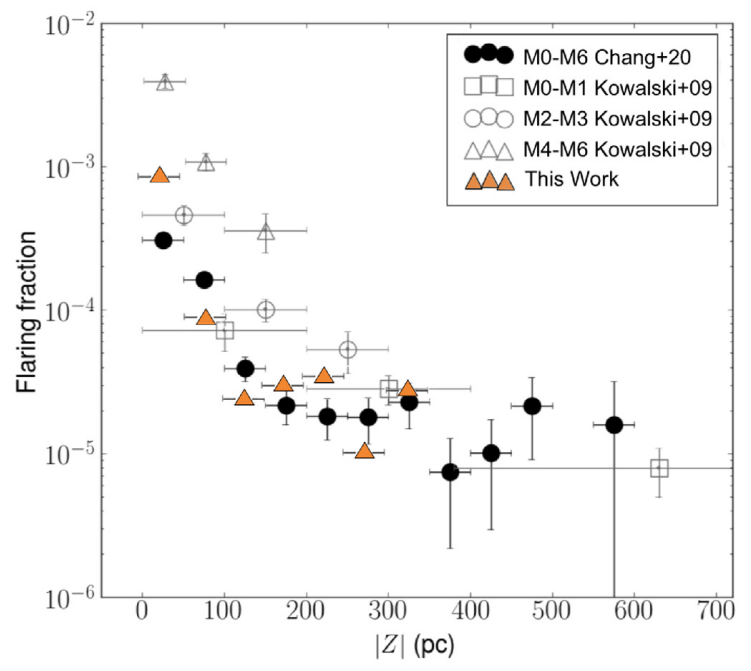

Figure 11. Fraction of flaring stars (normalized by total number of hours observed in this study) as a function of vertical distance from the Galactic plane $|Z|$ (orange triangles, $|Z|$ bin size $50 \mathrm{pc}$ ). We overlay results from Kowalski et al. (2009) for their spectral type ranges (M0-M1: open squares, M2-M3: open circles, M4-M6: open triangles) and from Chang et al. (2020) for their sample (M0-M6: closed circles).

\subsection{Flares from young stellar sources}

Stellar flare activity is known to decrease over stellar lifetimes. This occurs as a star loses angular momentum, via stellar winds, and consequently results in a quieting of the internal magnetic dynamo from decreased rotational velocity. This age-activity relationship has previously been directly connected with flare activity (e.g. Skumanich 1972; Wright et al. 2011; Davenport et al. 2019). Feinstein et al. (2020) studied young K5-M5 stars, and found that flare rates and amplitudes decreased for very young stars $\left(t_{\text {age }}>50 \mathrm{Myr}\right)$ across all temperatures $T_{\text {eff }} \geq 4000 \mathrm{~K}$.

We expect to find a broad distribution with higher average transverse velocity $V_{\mathrm{T}}$ across our full 500 pc sample, typical of the well studies age-velocity relationship within the Milky Way (Seabroke \& Gilmore 2007; Rix \& Bovy 2013; Mackereth et al. 2019). However, for the flare star sample of stars with low $Z$ positions, we expect these sources will have lower average $V_{\mathrm{T}}$ velocity, associated with a younger age. To investigate this age-activity relationship within our sample, we utilized Gaia DR2 proper motions to calculate the transverse velocity $\left(V_{\mathrm{T}}\right)$, in $\mathrm{km} \mathrm{s}^{-1}$, of the stars in our sample using,

$V_{\mathrm{T}}=4.74 \times\left(\mathrm{pmRA}^{2}+\mathrm{pmDEC}^{2}\right)^{0.5} \times D$,

where pmRA is the proper motion measured in the right ascension $\left(\right.$ mas $\left.\mathrm{yr}^{-1}\right)$, pmDEC is the proper motion measured in declination ( mas $^{-1}$ ), and $D$ is the distance to the source $(\mathrm{kpc})$.

Using $V_{\mathrm{T}}$, we can use the relationship between age and the observed transverse velocity of stellar sources within the Galactic thin disc.

To explore this, we calculated the cumulative distributions of transverse velocities across our full 500 pc sample of sources, and flaring sources, shown in Fig. 12. We find a median $V_{\mathrm{T}}$ of $35 \mathrm{~km} \mathrm{~s}^{-1}$ across our full $500 \mathrm{pc}$ sample of sources, and a median $V_{\mathrm{T}}$ of $24 \mathrm{~km} \mathrm{~s}^{-1}$ for the flaring sources only. When comparing the two cumulative distributions via the Kolmogorov-Smirnov test, we find a $p$-value of $<1$ per cent, suggesting two distinct distributions. Using the Gaia Universe Model Snapshot (GUMS) (Robin et al. 2012), we model the cumulative distribution of a full simulated $500 \mathrm{pc}$ sample 


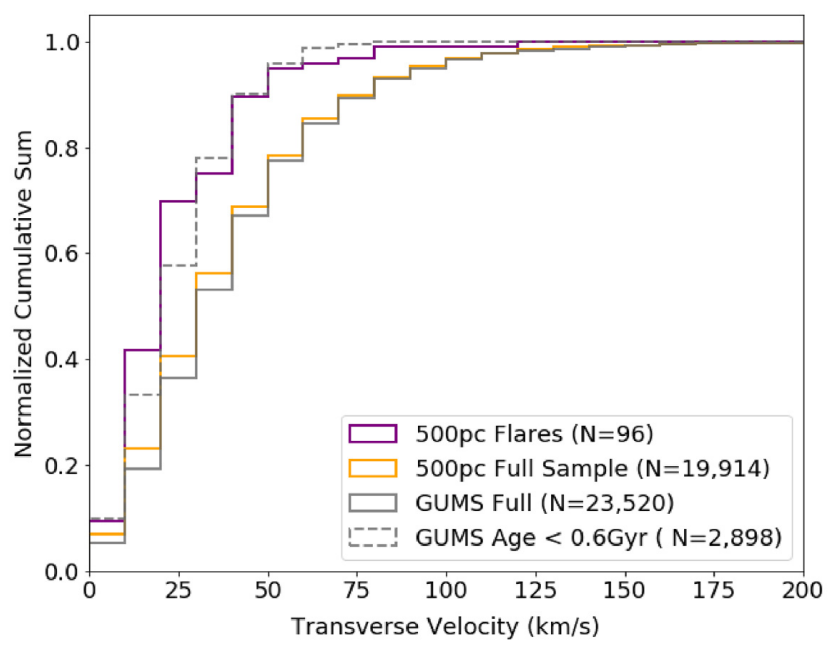

Figure 12. Cumulative distribution of transverse velocities for our $500 \mathrm{pc}$ flare sources (solid purple line), $500 \mathrm{pc}$ full sample of sources (solid orange line), GUMS $500 \mathrm{pc}$ sample (solid grey line), and 500 pc GUMS young sources $(<0.65 \mathrm{Gyr})$.

across our 12 fields and confirm that the distributions between the simulated and DWF full $500 \mathrm{pc}$ are nearly indistinguishable. We also present the cumulative distribution of isolated young sources $(<0.65 \mathrm{Gyr})$ from within the GUMS sample, which suggests a similar distribution to that of our flares.

From these results, we conclude that the majority of flaring sources within our sample are from young stellar sources within the thin disc.

\subsection{Short duration flare population}

We find a logarithmic distribution with a large duration range for our sample, as shown in panel (a) of Fig. 13. Specifically, we identify that the majority of flares in our sample occur on very short time-scales ( $\lesssim 8 \mathrm{~min}$ ). Note that the longest duration continuous light curve is 156 min on DWF field NGC 6744. The long flare durations are limited by our time on field (see Table 1) and by the criteria and means we use to identify flares (Section 3).

This sample of short duration flares contains sources across a broad range of flare amplitudes ( $\Delta M 0.1-1.8 \mathrm{mag}$ ), as well as across the full range of distances out to $500 \mathrm{pc}$, see panels (b) and (c) of Fig. 13. In Fig. 13(b), there are visible vertical lines along which flare durations cluster, an artefact of our continuous $20 \mathrm{~s}$ observations that cause the durations to artificially be distributed in multiples of $\sim 50$ $\mathrm{s}$ intervals ( $20 \mathrm{~s}$ exposures and $\sim 30 \mathrm{~s}$ readout). It is also important to note that the true flare duration values were not extrapolated for the cut-off flares in our sample (those with minimum duration times as a result of the limited time on field).

Short duration flares have previously been identified by Brasseur et al. (2019) within the GALEX (Bianchi \& GALEX Team 1999) mission's 10-s cadence data. The bulk of the stars observed by GALEX were within the mid-F to mid-K spectral type range. Our work confirms that this short duration population does indeed continue into the late- $\mathrm{K}$ to mid $\mathrm{M}$ dwarf stellar types, shown in Fig. 13(c).

Our sample of short duration flares spans a considerable energy range of $10^{31}-10^{34} \mathrm{erg}$, further diversifying the overall population of short duration flares beyond the GALEX sample. Interestingly, lower energy flares $\left(<10^{33} \mathrm{erg}\right)$ are not limited to only short duration events (see Fig. 7).
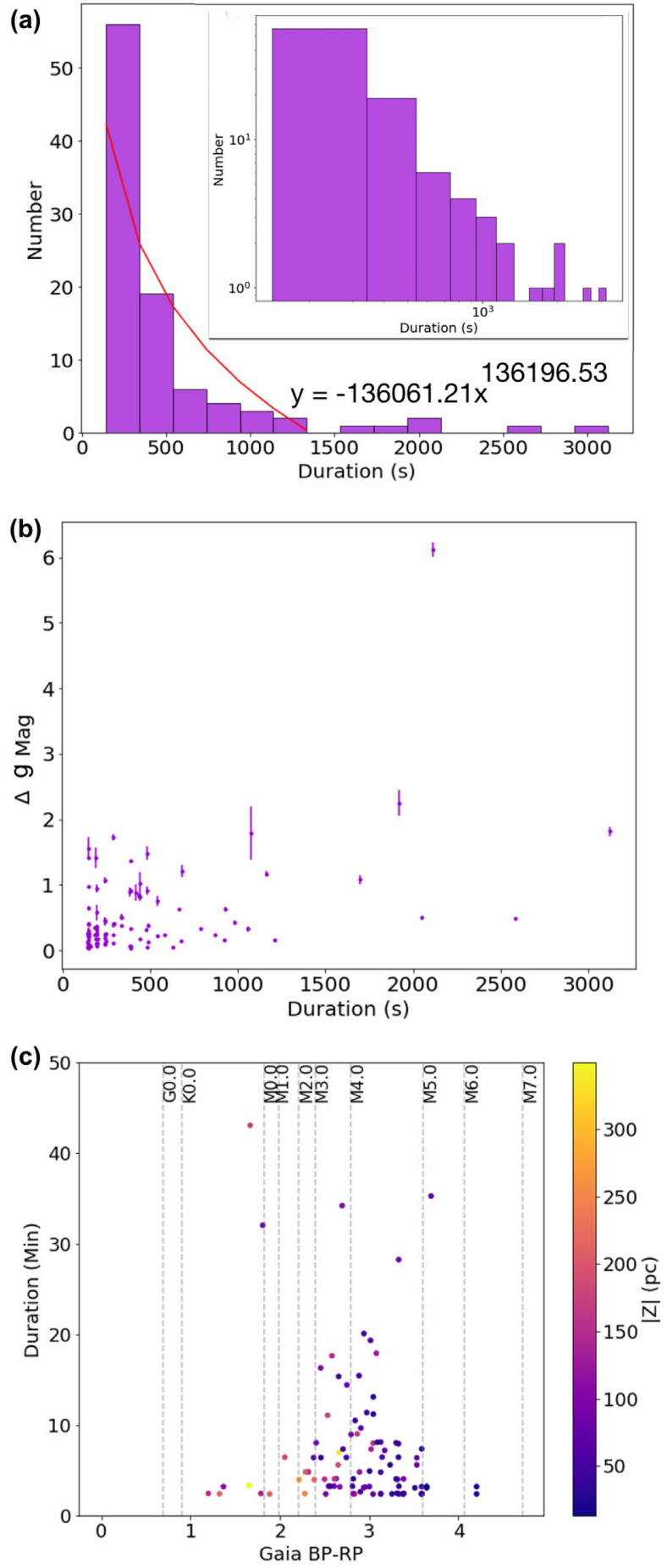

Figure 13. (a) Histogram of flare duration. The majority of flares occur over short durations, following an exponential relationship. (b) Change in magnitude (flare amplitude) versus duration, with short duration events spanning from 0.1-1.8 mag. The majority of flares have $<2$ ' $g$ '-band magnitude change, with one notable exception present at $\sim 6$ mag. (c) Duration (minutes) versus quiescent source colour (Gaia BP-RP). The colour of each point represents $|Z|$ (pc) as indicated by the colour bar. Short duration events are present across multiple spectral types out to $500 \mathrm{pc}$. 


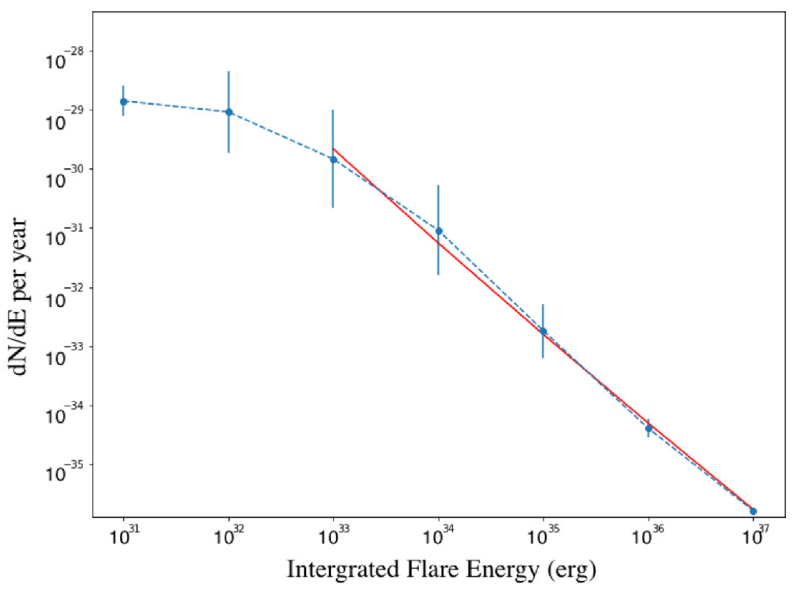

Figure 14. The FFD for all flares discovered in this work. The FFD spans the energy range of $10^{31}-10^{37}$. The error bar of each energy bin is calculated assuming Poisson statistics. The red line indicates the least-squares power law of $\log (\mathrm{d} N / \mathrm{d} E)$, finding $\alpha \sim 1.43 \pm 0.37$, fitted using data between $10^{33}$ and $10^{37}$.

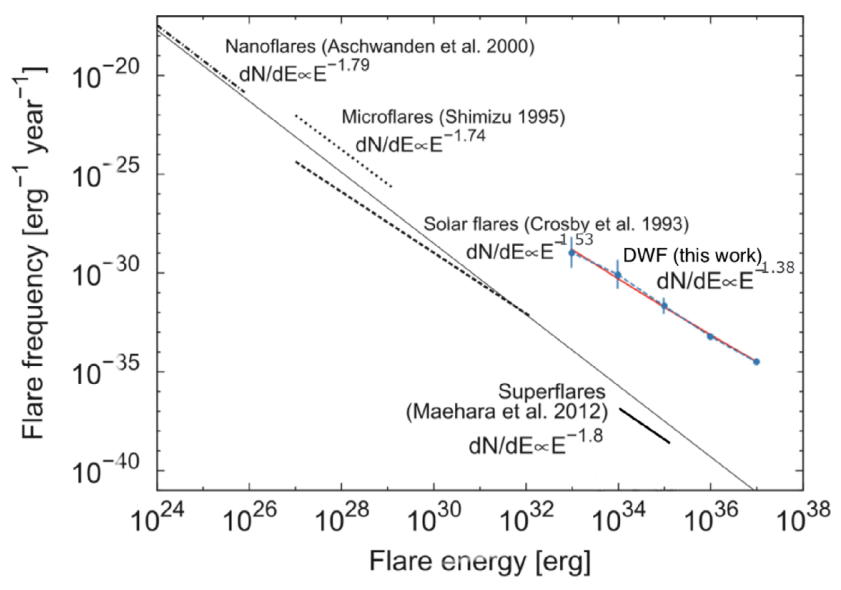

Figure 15. The FFD for the DWF sample of flares compared to several previous studies on solar-type flares.

\subsection{Flare frequency distribution}

The flare frequency distribution (FFD) represents the occurrence frequency $(\mathrm{d} N / \mathrm{d} E)$ of flares as a function of flare energy $(E)$, and can be expressed as a power-law relation, i.e. $\mathrm{d} N / \mathrm{d} E \sim E^{-\alpha}$. Previous work, including that on Solar flares and Kepler flares, find generally $\alpha$ is $\sim 2$, with slight variations in the value of $\alpha$ found across individual spectral types (Maehara et al. 2012; Shibata et al. 2013; Shibayama et al. 2013; Yang et al. 2017; Yang \& Liu 2019). For example, Audard et al. (2000) found that the FFD became flat towards later spectral types; however, Yang \& Liu (2019) findings indicate an increase in FFD across M dwarfs. Davenport et al. (2019) noted that comparing stellar types using specific flare rates generated from an individual FFD implicitly assumes that the flare rate for a star is governed by a single power law at all energies examined. This can be useful in the case of comparing samples with different observing conditions, however Davenport et al. (2019) further commented that in doing so we must ensure that a sufficient number of flares are observed in each spectral range to adequately measure the power law distribution within the FFD. With this in mind, we have not separated our sample to calculate FFDs for each individual spectral
Table 3. The number of flares and flaring sources recorded across all fields, with average hourly rates calculated based on the DECam $2.91 \mathrm{deg}^{2}$ field of view.

\begin{tabular}{lccccc}
\hline Field & Flares & $\begin{array}{c}\text { Flare } \\
\text { sources } \\
(\#)\end{array}$ & $\begin{array}{c}\text { Time } \\
\text { on field } \\
(\mathrm{h})\end{array}$ & $\begin{array}{c}\text { \# Flares } \\
\left(\mathrm{deg}^{-2} \mathrm{~h}^{-1}\right)\end{array}$ & $\begin{array}{c}\text { Galactic } \\
\text { latitude } \\
(\mathrm{deg})\end{array}$ \\
\hline Antlia & 8 & 8 & 7.03 & 0.39 & 19.172 \\
Dusty10 & 4 & 4 & 3.50 & 0.39 & -19.957 \\
Dusty12 & 3 & 3 & 4.39 & 0.23 & -21.889 \\
FRB131104 & 13 & 11 & 6.37 & 0.70 & -21.93 \\
8 h & 6 & 6 & 4.95 & 0.41 & -22.618 \\
Dusty11 & 1 & 1 & 2.35 & 0.14 & -22.814 \\
NGC 6744 & 27 & 18 & 18.38 & 0.50 & -26.054 \\
Prime & 16 & 16 & 6.88 & 0.80 & -30.262 \\
NSF2 & 3 & 3 & 3.61 & 0.28 & -39.823 \\
FRB010724 & 6 & 5 & 2.63 & 0.78 & -41.804 \\
4 h & 10 & 7 & 8.05 & 0.43 & -44.756 \\
3 h & 9 & 7 & 6.88 & 0.44 & -53.432 \\
\hline
\end{tabular}

Table 4. Flare rates determined for individual energy ranges with hourly and yearly rates calculate based on the DECam $2.91 \mathrm{deg}^{2}$ field of view.

\begin{tabular}{lccrrr}
\hline $\begin{array}{l}\text { Int. flare } \\
\text { energy } \\
\text { (erg) }\end{array}$ & \# Flares & $\begin{array}{c}\text { Rate } \\
\left(\mathrm{deg}^{-2} \mathrm{~h}^{-1}\right)\end{array}$ & $\begin{array}{c}\text { Rate } \\
\left(\mathrm{deg}^{-2} \mathrm{~d}^{-1}\right)\end{array}$ & $\begin{array}{c}\text { Rate } \\
\left(\mathrm{deg}^{-2} \mathrm{yr}^{-1}\right)\end{array}$ & $\begin{array}{c}\text { Rate } \\
\left(\mathrm{sky}^{-1} \mathrm{yr}^{-1}\right)\end{array}$ \\
\hline $10^{31}$ & 3 & 0.014 & 0.32 & 120.3 & $4.96 \times 10^{6}$ \\
$10^{32}$ & 20 & 0.091 & 2.19 & 801.9 & $3.31 \times 10^{7}$ \\
$10^{33}$ & 36 & 0.164 & 3.95 & 1443.4 & $5.95 \times 10^{7}$ \\
$10^{34}$ & 27 & 0.123 & 2.96 & 10082.6 & $4.47 \times 10^{7}$ \\
$10^{35}$ & 7 & 0.032 & 0.77 & 280.7 & $1.16 \times 10^{6}$ \\
$10^{36}$ & 2 & 0.009 & 0.21 & 80.2 & $3.31 \times 10^{6}$ \\
$10^{37}$ & 1 & 0.004 & 0.11 & 40.1 & $1.65 \times 10^{6}$ \\
Total & 96 & 0.43 & 10.54 & 3849 & $1.59 \times 10^{8}$ \\
\hline
\end{tabular}

types, rather calculating $\alpha$ from an FFD of the combined sample to ensure a sufficient number of flares in each energy bin. The FFD of the 96 flares in our sample, shown in Fig. 14, is fit with the least-squares power law of $\log (\mathrm{d} N / \mathrm{d} E)=E^{\alpha}$. We find a value of $\alpha \sim 1.43 \pm 0.37$, in agreement with previous $\alpha$ estimates across both solar-like and dwarf stars (e.g. Audard et al. 2000; Maehara et al. 2015; Yang \& Liu 2019), suggesting that the large portion of short duration flares found in this work are likely produced by the same physical dynamo mechanism. Our FFD does however suggest that higher energy flares occur less frequently amongst this paper's sample in comparison to previous work. Once the entirety of the archival DWF optical data is mined for flares, producing a full magnitude-limited catalogue, FFDs for each spectral type can be produced. This future work will assist with current research into understanding flare mechanisms between magnetically weak giants and magnetically active dwarf stars.

\subsection{Flare rates}

Across our 12 target fields and within $500 \mathrm{pc}$, we find that the number of flares per hour varies considerably, having an average of $0.5 \pm 0.2$ flares $\mathrm{deg}^{-2} \mathrm{~h}^{-1}$ (see Table 3). Our daily flare rate of 10.54 flares $\mathrm{deg}^{-2} \mathrm{~d}^{-1}$ is also comparable to the daily rate of 7.6 flares $\mathrm{deg}^{-2} \mathrm{~d}^{-1}$ on sources of $3500 \mathrm{~K} \leq T_{\text {eff }} \leq 4000 \mathrm{~K}$ across all flare energies from Feinstein et al. (2020).

We further breakdown flare rates across the range of integrated flare energies, as shown in Table 4 . We find the highest rates are associated with the lowest energy flares as expected and in agreement 
Table 5. Volumetic flare rates $\mathrm{pc}^{3}$ per hour. Calculated using $75.08 \mathrm{~h}$ of observations and a total observational volume of $443218 \mathrm{pc}^{3}$ for the 12 fields out to $500 \mathrm{pc}$.

\begin{tabular}{lcc}
\hline $\begin{array}{l}\Delta \text { g magnitude } \\
\text { of flare }\end{array}$ & $\begin{array}{c}\# \\
\text { Flares }\end{array}$ & $\begin{array}{c}\text { \# Flares } \\
\mathrm{pc}^{-3} \mathrm{~h}^{-1}\end{array}$ \\
\hline All & 96 & $2.88 \times 10^{-6}$ \\
$(0.0,0.5]$ & 65 & $1.90 \times 10^{-6}$ \\
$(0.5,1.0]$ & 16 & $4.81 \times 10^{-7}$ \\
$(1.0,1.5]$ & 9 & $2.70 \times 10^{-7}$ \\
$(1.5,2.0]$ & 4 & $1.20 \times 10^{-7}$ \\
$>2.0$ & 2 & $6.01 \times 10^{-8}$ \\
\hline
\end{tabular}

with previous studies (Maehara et al. 2012; Shibayama et al. 2013; Yang et al. 2017; Günther et al. 2020; Yang \& Liu 2019). Using the combined rates across all observed flare energies in this study, we estimate a sky rate of $\sim 0.43$ events $\mathrm{deg}^{-2} \mathrm{~h}^{-1}$ and volumetric rate of $\sim 2.9 \pm 0.3 \times 10^{-6}$ flares $\mathrm{pc}^{3} \mathrm{~h}^{-1}$, see Tables 3 and 5, respectively. Note: Our rates are likely an underestimate of the true flare rate, a reflection of selection criteria and observational limitations.

\section{CONCLUSIONS}

We present our 500 pc distance-limited stellar flare study, using DECam continuous $20 \mathrm{~s}$ cadence $g$-band data collected over 12 fields via the DWF. The advantage of our choice of continuous blue filter observations is our sensitivity to low-amplitude flares, where traditionally the detection efficiency drops at redder wavelengths. Furthermore, the unique observation strategy of DWF provides a 20$\mathrm{s}$ cadence sensitive to uncovering fast evolving short duration flares. We search the light curves of 19914 sources, each with multiple nightly light curves. The average time per visit was $\sim 74$ min on each $2.2^{\circ}$ diameter DECam field.

We find 96 flare events, occurring across 80 stars, from our volumelimited sample of 19914 sources. We found the following:

(i) Flares were found across K-M6 type stars, strongly concentrated around the mid-type $\mathrm{M}$ dwarfs.

(ii) The flares identified vary greatly in both duration and flare energy. Duration's span from $\sim 2.4$ to $\sim 52 \mathrm{~min}$, with $\sim 70$ per cent having duration's $<8 \mathrm{~min}$.

(iii) The short duration flares identified in this work span vast energy distribution of $10^{31}-10^{37} \mathrm{erg}$.

(iv) We confirm a strong relationship between flare energy and distance from the Galactic plane, with lower energy flares occurring $\sim<100 \mathrm{pc}$. This is consistent with the estimated scale height of the Galactic young disc.

(v) The flaring fraction of stars is also identified to decrease with vertical distance from the Galactic plane, in agreement with previous studies.

(vi) We determine, using our sources transverse velocity, that our flares are generated from likely young stellar sources.

(vii) Our FFD confirms a power law relationship between flare number and flare energy, and generates a value of $\alpha$ is consistent with previous work.

(viii) We determine an average flare rate of $0.42 \pm 0.2$ flares deg ${ }^{-2}$ $\mathrm{h}^{-1}$, and a volumetric rate of $2.9 \pm 0.3 \times 10^{-6}$ flares $\mathrm{pc}^{-3} \mathrm{~h}^{-1}$.

We plan to continue to explore flare activity within the DWF optical data sets, aiming to provide a full magnitude-limited flare catalog. DWF will also aim to explore multiwavelength properties of flares via past and future coordinated DWF simultaneous observations.

\section{ACKNOWLEDGEMENTS}

We would like to acknowledge and thank our reviewer for their very insight and helpful review and comments. Part of this research was funded by the Australian Research Council Centre of Excellence for Gravitational Wave Discovery (OzGrav), CE170100004. JC acknowledges funding by the Australian Research Council Discovery Project, DP200102102. We acknowledge the financial assistance of the National Research Foundation (NRF). Opinions expressed and conclusions arrived at, are those of the authors and are not necessarily to be attributed to the NRF. This work was partly supported by the GROWTH (Global Relay of Observatories Watching Transients Happen) project funded by the National Science Foundation under PIRE Grant No. 1545949. SAB acknowledges support from the Aliyun Fellowship and Chinese Academy of Sciences President's International Fellowship Initiative Grant. This work has made use of data from the European Space Agency (ESA) mission Gaia (https: //www.cosmos.esa. int/gaia), processed by the Gaia Data Processing and Analysis Consortium (DPAC, https://www.cosmos.esa. int/web /gaia/dpac/consortium). Funding for the DPAC has been provided by national institutions, in particular the institutions participating in the Gaia Multilateral Agreement. This publication makes use of data products from the Wide-field Infrared Survey Explorer, which is a joint project of the University of California, Los Angeles, and the Jet Propulsion Laboratory/California Institute of Technology, funded by the National Aeronautics and Space Administration.

This project used data obtained with the Dark Energy Camera (DECam), which was constructed by the Dark Energy Survey (DES) collaboration. Funding for the DES Projects has been provided by the U.S. Department of Energy, the U.S. National Science Foundation, the Ministry of Science and Education of Spain, the Science and Technology Facilities Council of the United Kingdom, the Higher Education Funding Council for England, the National Center for Supercomputing Applications at the University of Illinois at Urbana-Champaign, the Kavli Institute of Cosmological Physics at the University of Chicago, the Center for Cosmology and AstroParticle Physics at the Ohio State University, the Mitchell Institute for Fundamental Physics and Astronomy at Texas A\&M University, Financiadora de Estudos e Projetos, Fundação Carlos Chagas Filho de Amparo à Pesquisa do Estado do Rio de Janeiro, Conselho Nacional de Desenvolvimento Científico e Tecnológico and the Ministério da Ciência, Tecnologia e Inovacão, the Deutsche Forschungsgemeinschaft, and the Collaborating Institutions in the Dark Energy Survey. The Collaborating Institutions are Argonne National Laboratory, the University of California at Santa Cruz, the University of Cambridge, Centro de Investigaciones Enérgeticas, Medioambientales y Tecnológicas-Madrid, the University of Chicago, University College London, the DES-Brazil Consortium, the University of Edinburgh, the Eidgenössische Technische Hochschule (ETH) Zürich, Fermi National Accelerator Laboratory, the University of Illinois at UrbanaChampaign, the Institut de Ciències de l'Espai (IEEC/CSIC), the Institut de Física d'Altes Energies, Lawrence Berkeley National Laboratory, the Ludwig-Maximilians Universität München and the associated Excellence Cluster Universe, the University of Michigan, the National Optical Astronomy Observatory, the University of Nottingham, the Ohio State University, the OzDES Membership Consortium the University of Pennsylvania, the University of Portsmouth, SLAC National Accelerator Laboratory, Stanford University, the University of Sussex, and Texas A\&M University.

This study is based on observations at Cerro Tololo InterAmerican Observatory, National Optical Astronomy Observatory which is operated by the Association of Universities for Research 
in Astronomy (AURA) under a cooperative agreement with the National Science Foundation. SW and SG would like to thank their greyhound Lucy for being a great listener to science conversations, and eater of all the treats.

\section{DATA AVAILABILITY}

The data underlying this article will be shared on reasonable request to the corresponding author.

\section{REFERENCES}

Abazajian K. N. et al., 2009, ApJS, 182, 543

Andreoni I., Cooke J., 2019, IAUS, 339, 135

Andreoni I. et al., 2020, MNRAS, 491, 5852

Audard M., Güdel M., Drake J. J., Kashyap V. L., 2000, ApJ, 541, 396

Berger E. et al., 2008, ApJ, 690, 231

Bertin E., Arnouts S., 1996, A\&AS, 117, 393

Bertin E., Arnouts S., 2010, Astrophysics Source Code Library, record ascl: 1010.064

Bianchi L., GALEX Team, 1999, Mem. Soc. Astron. Italiana, 70, 365

Binney J., Tremaine S., 1987, Galactic Dynamics, Princeton Univ. Press

Bopp B. W., Moffett T. J., 1973, ApJ, 185, 239

Borucki W. J. et al., 2010, Science, 327, 977

Brasseur C. E., Osten R. A., Fleming S. W., 2019, ApJ, 883, 88

Cabrera-Lavers A., Bilir S., Ak S., Yaz E., López-Corredoira M., 2007, A\&A, 464,565

Casagrande L. et al., 2020, preprint (arXiv:2011.02517)

Chabrier G., Baraffe I., 1997, A\&A, 327, 1039

Chang S. W., Byun Y. I., Hartman J. D., 2015, ApJ, 814, 35

Chang S.-W., Wolf C., Onken C. A., 2020, MNRAS, 491, 39

Cutri R. M. et al., 2021, VizieR Online Data Catalog. p. II/328

Dal H. A., Evren S., 2010, AJ, 140, 483

Davenport J. R. A., 2016, in Kosovichev A. G., Hawley S. L., Heinzel P., eds, Proc. IAU Symp. 320, Solar and Stellar Flares and their Effects on Planets. Honolulu, United States,p. 128

Davenport J. R. A., Becker A. C., Kowalski A. F., Hawley S. L., Schmidt S. J., Hilton E. J., Sesar B., Cutri R., 2012, ApJ, 748, 58

Davenport J. R. A., Covey K. R., Clarke R. W., Boeck A. C., Cornet J., Hawley S. L., 2019, ApJ, 871, 241

Dillon C. J. et al., 2020, ApJ, 904, 109

Dorman B., Nelson L. A., Chau W. Y., 1989, ApJ, 342, 1003

Ducati J. R., Bevilacqua C. M., Rembold S. B., Ribeiro D., 2001, ApJ, 558, 309

Evans D. W. et al., 2018, A\&A, 616, A4

Feinstein A. D., Montet B. T., Ansdell M., Nord B., Bean J. L., Günther M. N., Gully-Santiago M. A., Schlieder J. E., 2020, AJ, 160, 219

Flaugher B. et al., 2015, AJ, 150, 150

Furusawa H. et al., 2018, PASJ, 70, S3

Garnavich P. M. et al., 2016, ApJ, 820, 23

Günther M. N. et al., 2020, AJ, 159, 60

Haisch B., Strong K. T., Rodono M., 1991, ARA\&A, 29, 275

Hawley S. L., Pettersen B. R., 1991, ApJ, 378, 725

Hawley S. L., Davenport J. R. A., Kowalski A. F., Wisniewski J. P., Hebb L., Deitrick R., Hilton E. J., 2014, ApJ, 797, 121

Hilton E. J. et al., 2010, AJ, 140, 1402

Howard W. S. et al., 2018, ApJ, 860, L30

Howard W. S. et al., 2020, ApJ, 902, 115

Ishida K., Ichimura K., Shimizu Y., Mahasenaputra, 1991, Ap\&SS, 182, 227

Jackman J. A. G. et al., 2018, MNRAS, 477, 4655

Jurić M. et al., 2008, ApJ, 673, 864

Kawanomoto S. et al., 2018, PASJ, 70, 66

Komiyama Y. et al., 2018, PASJ, 70, S2

Kowalski A. F. et al., 2009, AJ, 138, 633

Lacy C. H., Moffett T. J., Evans D. S., 1976, ApJS, 30, 85

Law N. M., Fors O., Wulfken P., Ratzloff J., Kavanaugh D., 2014, in Stepp L. M., Gilmozzi R., Hall H. J., eds, Proc. SPIE Conf. Ser. Vol. 9145, Ground-Based and Airborne Telescopes V. SPIE, Bellingham, p. $91450 Z$
Lin R. P., Hudson H. S., 1976, Sol. Phys., 50, 153

Lochner M., Bassett B. A., 2020, Astron. Comput., 36, 100481

Luri X. et al., 2018, A\&A, 616, A9

Mackereth J. T. et al., 2019, MNRAS, 489, 176

Maehara H. et al., 2012, Nature, 485, 478

Maehara H., Shibayama T., Notsu Y., Notsu S., Honda S., Nogami D., Shibata K., 2015, Earth Planets Space, 67, 59

Miyazaki S. et al., 2018, PASJ, 70, S1

Moffett T. J., 1974, ApJS, 29, 1

Mondrik N., Newton E., Charbonneau D., Irwin J., 2019, ApJ, 870, 10

Morales J. C. et al., 2009, ApJ, 691, 1400

Mould J., Clementini G., Da Costa G., 2019, Publ. Astron. Soc. Aust., 36, $\mathrm{e} 001$

Namekata K. et al., 2017, ApJ, 851, 91

Onken C. A. et al., 2019, Publ. Astron. Soc. Aust., 36, e033

Osten R. A., Wolk S. J., 2015, ApJ, 809, 79

Osten R. A. et al., 2005, ApJ, 621, 398

Parnell C. E., Jupp P. E., 2000, ApJ, 529, 554

Perley D. A. et al., 2018, MNRAS, 484, 1031

Pettersen B. R., 1989, Sol. Phys., 121, 299

Pineda J. S. et al., 2013, AJ, 146, 50

Prentice S. J. et al., 2018, ApJ, 865, L3

Ricker G. R. et al., 2009, Bulletin of the American Astronomical Society, 41, 193

Rix H.-W., Bovy J., 2013, A\&AR, 21, 61

Robin A. C. et al., 2012, A\&A, 543, A100

Rodríguez Martínez R., Lopez L. A., Shappee B. J., Schmidt S. J., Jayasinghe T., Kochanek C. S., Auchettl K., Holoien T. W. S., 2020a, ApJ, 892, 144

Rodríguez Martínez R., Lopez L. A., Shappee B. J., Schmidt S. J., Jayasinghe T., Kochanek C. S., Auchettl K., Holoien T. W. S., 2020b, ApJ, 892, 144

Schmidt S. J., Wagoner E. L., Johnson J. A., Davenport J. R. A., Stassun K. G., Souto D., Ge J., 2016, MNRAS, 460, 2611

Schmidt S. J. et al., 2018, ApJ, 876, 115

Scott D., Pierfederici F., Swaters R. A., Thomas B., Valdes F. G., 2007, in Shaw R. A., Hill F., Bell D. J., eds, ASP Conf. Ser. Vol. 376, Astronomical Data Analysis Software and Systems XVI. Astron. Soc. Pac., San Francisco, p. 265

Seabroke G. M., Gilmore G., 2007, MNRAS, 380, 1348

Shappee B. et al., 2012, in American Astronomical Society Meeting Abstracts \#220. p. 432.03

Shibata K. et al., 2013, PASJ, 65, 49

Shibayama T. et al., 2013, ApJS, 209, 5

Skumanich A., 1972, ApJ, 171, 565

Stelzer B., Damasso M., Scholz A., Matt S. P., 2016, MNRAS, 463, 1844

Swaters R. A., Valdes F. G., 2007, in Shaw R. A., Hill F., Bell D. J., eds, ASP Conf. Ser. Vol. 376, Astronomical Data Analysis Software and Systems XVI. Astron. Soc. Pac., San Francisco, p. 269

Ting K., Liu F., Zhou Z., 2008, in ICDM 2008. Eighth IEEE International Conference on Data Mining. IEEE Computer Society, Los Alamitos, CA, USA, p. 413

Trumpler R. J., Weaver H. F., 1953, in Trumpler R. J., Weaver H. F., eds, Statistical Astronomy. University of California Press, Berkeley

Valdes F. G., Swaters R. A., 2007, in Shaw R. A., Hill F., Bell D. J., eds, ASP Conf. Ser. Vol. 376, Astronomical Data Analysis Software and Systems XVI. Astron. Soc. Pac., San Francisco, p. 273

Van Doorsselaere T., Shariati H., Debosscher J., 2017, ApJS, 232, 26

Walkowicz L. M. et al., 2011, AJ, 141, 50

Webb S. et al., 2020, MNRAS, 498, 3077

West A. A., Hawley S. L., 2008, PASP, 120, 1161

West A. A. et al., 2004, AJ, 128, 426

West A. A., Hawley S. L., Bochanski J. J., Covey K. R., Reid I. N., Dhital S., Hilton E. J., Masuda M., 2008, AJ, 135, 785

Wheatley P. J. et al., 2018, MNRAS, 475, 4476

Wolf C. et al., 2018, Publ. Astron. Soc. Aust., 35, e010

Wright N. J., Drake J. J., Mamajek E. E., Henry G. W., 2011, ApJ, 743, 48

Yang H., Liu J., 2019, ApJS, 241, 29

Yang H. et al., 2017, ApJ, 849, 36 
APPENDIX A: DWF 500 PC FLARES

Table A1. DWF 500 pc flare sample properties.

\begin{tabular}{|c|c|c|c|c|c|c|c|c|c|c|c|c|}
\hline Date & Gaia DR2 ID & $\begin{array}{l}\text { Quies- } \\
\text {-cent } \\
\text { mag } \\
(g)\end{array}$ & $\begin{array}{c}\text { Gaia } \\
\mathrm{BP}-\mathrm{RP}\end{array}$ & $\begin{array}{c}\text { WISE } \\
W 1-W 2\end{array}$ & $\begin{array}{c}\mathrm{SM} \mathrm{DR} 2 / 3 \\
r-z\end{array}$ & $\begin{array}{l}\text { Flare } \\
\Delta \\
\text { mag } \\
(g)\end{array}$ & $\begin{array}{l}\text { Flare } \\
\text { Dura- } \\
\text {-tion }{ }^{a} \\
\text { (s) }\end{array}$ & $\begin{array}{l}\text { Dist- } \\
\text {-ance } \\
\text { (pc) }\end{array}$ & $\begin{array}{c}\text { Quies- } \\
\text {-cent } \\
\text { flux } \\
\left(\text { erg s}^{-1}\right)\end{array}$ & $\begin{array}{l}\text { Rela- } \\
\text {-tive } \\
\text { flux } \\
\left(\sum\right)\end{array}$ & $\begin{array}{c}\text { Flare } \\
\text { energy }^{b} \\
\text { ( } g \text { band) } \\
\text { (erg) }\end{array}$ & $\begin{array}{c}\text { Flare } \\
\text { energy }^{b} \\
(\text { bol) } \\
(\mathrm{erg})\end{array}$ \\
\hline 151218 & 4636450838513006208 & 17.63 & 2.95 & 0.19 & 2.57 & 0.17 & 189.48 & 73.80 & $1.25 \times 10^{30}$ & 0.32 & $7.51 \times 10^{31}$ & $6.91 \times 10^{32}$ \\
\hline 151222 & 4636433108887748864 & 17.26 & 3.23 & 0.19 & 2.75 & 0.38 & 336.35 & 39.90 & $5.39 \times 10^{29}$ & 0.80 & $1.45 \times 10^{32}$ & $1.33 \times 10^{33}$ \\
\hline 151218 & 4636360850358017792 & 17.61 & 2.94 & 0.20 & 2.48 & 0.16 & $1206.85+$ & 61.94 & $9.48 \times 10^{29}$ & 1.41 & $1.61 \times 10^{33}+$ & $1.48 \times 10^{34}$ \\
\hline 151219 & 4637865394582180480 & 16.44 & 3.33 & 0.17 & 2.90 & 1.36 & 476.49 & 50.75 & $1.85 \times 10^{30}$ & 0.80 & $6.48 \times 10^{32}$ & $5.96 \times 10^{33}$ \\
\hline 151220 & 4637865394582180480 & 16.44 & 3.33 & 0.17 & 2.90 & 1.36 & 386.81 & 50.75 & $1.85 \times 10^{30}$ & 4.04 & $2.89 \times 10^{33}$ & $2.66 \times 10^{34}$ \\
\hline 151218 & 4728699589203817600 & 18.37 & 3.13 & 0.21 & 2.52 & 0.25 & 144.22 & 58.26 & $3.85 \times 10^{29}$ & 0.22 & $1.23 \times 10^{31}$ & $1.13 \times 10^{32}$ \\
\hline 151218 & 4728699589203817600 & 18.37 & 3.13 & 0.21 & 2.52 & 0.24 & $287.72+$ & 58.26 & $3.85 \times 10^{29}$ & 0.53 & $5.87 \times 10^{31}+$ & $5.40 \times 10^{32}$ \\
\hline 151221 & 4728699589203817600 & 18.39 & 3.13 & 0.21 & 2.52 & 0.64 & 144.41 & 58.26 & $3.82 \times 10^{29}$ & 1.06 & $5.89 \times 10^{31}$ & $5.42 \times 10^{32}$ \\
\hline 151222 & 4728699589203817600 & 18.36 & 3.13 & 0.21 & 2.52 & 0.38 & 487.09 & 58.26 & $4.17 \times 10^{29}$ & 1.34 & $2.57 \times 10^{32}$ & $2.37 \times 10^{33}$ \\
\hline 151218 & 4728703055241994752 & 20.94 & 3.69 & 0.17 & 3.61 & 6.12 & $2115.37+$ & 97.35 & $1.08 \times 10^{29}$ & 1105.8 & $2.38 \times 10^{35}+$ & $2.19 \times 10^{36}$ \\
\hline 151219 & 4734505525995537024 & 18.03 & 3.32 & 0.18 & 2.80 & 0.09 & 240.32 & 47.31 & $3.73 \times 10^{29}$ & 0.15 & $1.35 \times 10^{31}$ & $1.24 \times 10^{32}$ \\
\hline 151220 & 4683390089413218688 & 19.93 & 4.20 & 0.23 & 3.32 & 0.97 & 143.69 & 38.54 & $2.74 \times 10^{29}$ & 0.33 & $2.02 \times 10^{32}$ & $1.85 \times 10^{33}$ \\
\hline 151220 & 4683390089413218688 & 19.93 & 4.20 & 0.23 & 3.32 & 0.94 & 192.29 & 38.54 & $2.74 \times 10^{29}$ & 2.01 & $1.65 \times 10^{31}$ & $1.52 \times 10^{32}$ \\
\hline 150114 & 4779473318188791680 & 16.84 & 3.05 & 0.20 & 2.51 & 0.14 & 672.56 & 38.51 & $7.38 \times 10^{29}$ & 0.33 & $1.64 \times 10^{32}$ & $1.51 \times 10^{33}$ \\
\hline 150117 & 4779473318188791680 & 16.84 & 3.05 & 0.20 & 2.51 & 0.33 & 786.71 & 38.51 & $7.38 \times 10^{29}$ & 0.91 & $4.95 \times 10^{32}$ & $4.55 \times 10^{33}$ \\
\hline 151221 & 4779669134337892864 & 18.85 & 3.05 & 0.19 & 2.49 & 0.13 & 142.84 & 97.66 & $7.52 \times 10^{29}$ & 0.21 & $2.06 \times 10^{31}$ & $1.90 \times 10^{32}$ \\
\hline 170205 & 5502472749699352320 & 17.17 & 2.82 & 0.18 & 2.28 & 0.14 & 243.49 & 75.21 & $3.07 \times 10^{30}$ & 0.31 & $1.56 \times 10^{32}$ & $1.44 \times 10^{33}$ \\
\hline 150114 & 5502105963787924480 & 17.98 & 3.64 & 0.22 & 3.16 & 0.09 & 193.21 & 34.35 & $1.94 \times 10^{29}$ & 0.19 & $7.11 \times 10^{30}$ & $6.54 \times 10^{31}$ \\
\hline 150117 & 5502105963787924480 & 17.98 & 3.64 & 0.22 & 3.16 & 0.35 & 184.04 & 34.35 & $1.94 \times 10^{29}$ & 0.56 & $2.01 \times 10^{31}$ & $1.85 \times 10^{32}$ \\
\hline 170203 & 5447196146939389568 & 19.31 & 3.09 & 0.19 & 2.57 & 0.12 & 485.75 & 81.13 & $3.37 \times 10^{29}$ & 0.60 & $9.84 \times 10^{31}$ & $9.05 \times 10^{32}$ \\
\hline 170206 & 5444145826805790080 & 19.12 & 3.31 & 0.21 & 2.70 & 0.10 & 147.62 & 98.85 & $5.60 \times 10^{29}$ & 3.16 & $2.62 \times 10^{32}$ & $2.41 \times 10^{33}$ \\
\hline 160802 & 6435079169511271040 & 19.78 & 3.30 & 0.18 & 2.89 & 0.91 & 482.12 & 97.22 & $2.89 \times 10^{29}$ & 0.16 & $2.30 \times 10^{31}$ & $2.11 \times 10^{32}$ \\
\hline 160803 & 6435079169511271040 & 19.92 & 3.30 & 0.18 & 2.89 & 0.47 & 242.23 & 97.22 & $2.89 \times 10^{29}$ & 1.01 & $7.08 \times 10^{31}$ & $6.51 \times 10^{32}$ \\
\hline 160803 & 6438591692548589056 & 18.91 & 3.33 & 0.25 & 2.80 & 0.07 & 194.56 & 85.74 & $1.18 \times 10^{29}$ & 0.20 & $2.14 \times 10^{31}$ & $1.97 \times 10^{32}$ \\
\hline 160804 & 6438591692548589056 & 18.91 & 3.33 & 0.25 & 2.80 & 0.21 & 144.22 & 85.74 & $1.18 \times 10^{29}$ & 0.39 & $1.25 \times 10^{32}$ & $1.15 \times 10^{33}$ \\
\hline 160728 & 6435402765228918144 & 20.00 & 3.59 & 0.23 & 3.12 & 0.22 & 143.77 & 80.42 & $1.72 \times 10^{29}$ & 1.08 & $2.68 \times 10^{31}$ & $2.47 \times 10^{32}$ \\
\hline 160802 & 6435402765228918144 & 20.05 & 3.59 & 0.23 & 3.12 & 0.36 & 192.16 & 80.41 & $1.72 \times 10^{29}$ & 0.58 & $1.94 \times 10^{31}$ & $1.78 \times 10^{32}$ \\
\hline 160802 & 6435402765228918144 & 20.05 & 3.59 & 0.23 & 3.12 & 0.22 & 144.37 & 80.41 & $1.72 \times 10^{29}$ & 0.41 & $1.01 \times 10^{31}$ & $9.31 \times 10^{31}$ \\
\hline 160803 & 6435402765228918144 & 20.05 & 3.59 & 0.23 & 3.12 & 0.30 & 144.63 & 80.41 & $1.72 \times 10^{29}$ & 0.51 & $1.05 \times 10^{31}$ & $1.39 \times 10^{33}$ \\
\hline 160807 & 6435402765228918144 & 20.05 & 3.59 & 0.23 & 3.12 & 0.81 & 442.08 & 80.41 & $1.72 \times 10^{29}$ & 3.35 & $2.55 \times 10^{32}$ & $2.34 \times 10^{33}$ \\
\hline 160804 & 6435402700808342528 & 14.71 & 2.46 & 0.11 & 1.87 & 0.04 & 384.66 & 79.89 & $2.26 \times 10^{31}$ & 0.22 & $1.94 \times 10^{33}$ & $1.78 \times 10^{33}$ \\
\hline 160805 & 6435404453155002624 & 18.94 & 3.38 & 0.21 & 3.00 & 0.41 & 144.12 & 94.09 & $6.37 \times 10^{29}$ & 0.90 & $8.27 \times 10^{31}$ & $7.60 \times 10^{32}$ \\
\hline 160805 & 6435404453155002624 & 18.94 & 3.38 & 0.21 & 3.00 & 1.41 & 143.94 & 94.09 & $6.37 \times 10^{29}$ & 3.59 & $3.29 \times 10^{32}$ & $3.03 \times 10^{33}$ \\
\hline 160806 & 6438625781704284928 & 16.75 & 2.84 & 0.18 & 2.33 & 0.06 & 629.72 & 94.79 & $4.90 \times 10^{30}$ & 0.41 & $1.26 \times 10^{33}$ & $1.16 \times 10^{34}$ \\
\hline 150117 & 4758284595250930560 & 19.09 & 3.51 & 0.19 & 2.95 & 0.25 & 183.69 & 60.58 & $2.15 \times 10^{29}$ & 0.44 & $1.76 \times 10^{31}$ & $1.61 \times 10^{32}$ \\
\hline 170202 & 4758503569863047424 & 16.03 & 2.66 & 0.17 & 2.09 & 0.17 & $922.50+$ & 96.63 & $9.22 \times 10^{30}$ & 0.96 & $8.17 \times 10^{33}+$ & $7.52 \times 10^{34}$ \\
\hline 170202 & 4758620770931164544 & 17.63 & 2.90 & 0.16 & 2.37 & 0.07 & 157.89 & 89.61 & $1.81 \times 10^{30}$ & 0.10 & $2.97 \times 10^{31}$ & $2.74 \times 10^{32}$ \\
\hline 160730 & 5191257877539468672 & 17.41 & 3.25 & 0.23 & 2.75 & 0.08 & 144.29 & 69.57 & $1.42 \times 10^{30}$ & 0.11 & $2.15 \times 10^{31}$ & $1.98 \times 10^{32}$ \\
\hline 151218 & 4728688628448657280 & 17.69 & 2.51 & 0.17 & 1.88 & 0.25 & 143.49 & 113.05 & $2.74 \times 10^{30}$ & 0.29 & $1.17 \times 10^{32}$ & $1.07 \times 10^{33}$ \\
\hline 151222 & 4728701135393440384 & 16.62 & 1.65 & -0.04 & 0.82 & 0.18 & 200 & 435.55 & $1.09 \times 10^{32}$ & 0.21 & $4.55 \times 10^{33}$ & $4.19 \times 10^{34}$ \\
\hline 151219 & 4683671426951508864 & 18.61 & 3.53 & 0.21 & 3.10 & 0.51 & 336.54 & 108.14 & $1.07 \times 10^{30}$ & 1.85 & $6.67 \times 10^{32}$ & $4.38 \times 10^{33}$ \\
\hline 150116 & 4683671426951508864 & 18.61 & 3.53 & 0.21 & 3.10 & 0.89 & 383.17 & 108.14 & $1.07 \times 10^{30}$ & 3.04 & $4.76 \times 10^{32}$ & $6.14 \times 10^{33}$ \\
\hline 150114 & 4683434958935655552 & 22.19 & 2.66 & 0.26 & 2.40 & 0.88 & 418.77 & 476.23 & $7.70 \times 10^{29}$ & 2.78 & $8.98 \times 10^{32}$ & $8.26 \times 10^{33}$ \\
\hline 150115 & 4779763005142093696 & 18.62 & 2.21 & 0.14 & 1.35 & 0.1 & 237.83 & 384.70 & $1.35 \times 10^{31}$ & 0.28 & $9.24 \times 10^{32}$ & $8.50 \times 10^{33}$ \\
\hline 150116 & 4778723996718806784 & 20.57 & 2.86 & 0.18 & 2.44 & 0.75 & 541.34 & 233.38 & $8.23 \times 10^{29}$ & 1.53 & $6.81 \times 10^{32}$ & $6.27 \times 10^{33}$ \\
\hline 180606 & 5209798289282962048 & 15.71 & 1.66 & 0.03 & 0.94 & 0.49 & 2584.01 & 453.82 & $2.73 \times 10^{32}$ & 13.27 & $9.37 \times 10^{36}$ & $8.62 \times 10^{37}$ \\
\hline 180606 & 5209089821541031680 & 18.74 & 2.54 & 0.16 & 1.93 & 0.27 & 195.22 & 287.84 & $6.75 \times 10^{30}$ & 0.34 & $4.52 \times 10^{32}$ & $4.16 \times 10^{33}$ \\
\hline 180609 & 5209799457513998720 & 17.53 & 2.81 & 0.19 & 2.29 & 0.23 & 195.16 & 101.59 & $2.56 \times 10^{30}$ & 0.58 & $2.92 \times 10^{32}$ & $2.69 \times 10^{33}$ \\
\hline 180609 & 5209096246811787904 & 22.06 & 3.08 & 0.13 & 2.42 & 1.79 & 1075.85 & 328.14 & $4.12 \times 10^{29}$ & 10.20 & $4.52 \times 10^{33}$ & $4.16 \times 10^{34}$ \\
\hline 180606 & 5209076425539832448 & 19.59 & - & 0.16 & 2.25 & 1.82 & 3121.58 & 404.49 & $6.09 \times 10^{30}$ & 51.91 & $9.87 \times 10^{35}$ & $9.08 \times 10^{36}$ \\
\hline 170203 & 5447129489047169536 & 19.14 & 2.79 & 0.13 & 2.36 & 0.22 & 537.92 & 263.78 & $3.92 \times 10^{30}$ & 1.41 & $2.98 \times 10^{33}$ & $2.74 \times 10^{34}$ \\
\hline 180608 & 5444073018520551296 & 19.46 & 3.14 & 0.23 & 2.67 & 0.91 & 387.53 & 173.67 & $1.27 \times 10^{30}$ & 1.89 & $9.28 \times 10^{32}$ & $8.54 \times 10^{33}$ \\
\hline 170203 & 5444224407527438208 & 17.21 & 1.79 & -0.01 & 0.93 & 0.04 & 146.33 & 423.10 & $5.97 \times 10^{31}$ & 0.07 & $6.45 \times 10^{32}$ & $5.93 \times 10^{33}$ \\
\hline 170207 & 5445901884314615680 & 18.87 & 2.46 & 0.11 & 1.86 & 0.43 & 978.76 & 323.55 & $7.57 \times 10^{30}$ & 2.89 & $2.14 \times 10^{34}$ & $1.97 \times 10^{35}$ \\
\hline 170205 & 5444220314420785280 & 20.13 & 2.60 & 0.21 & 2.09 & 0.58 & 194.93 & 232.57 & $1.22 \times 10^{30}$ & 1.94 & $4.64 \times 10^{32}$ & $4.27 \times 10^{33}$ \\
\hline 170205 & 5445595601606963200 & 17.23 & 2.63 & 0.12 & 2.05 & 0.25 & 245.18 & 201.15 & $1.32 \times 10^{30}$ & 0.31 & $9.93 \times 10^{32}$ & $9.14 \times 10^{33}$ \\
\hline 180609 & 5201723441529787392 & 19.12 & 2.97 & 0.19 & 2.36 & 1.21 & 682.82 & 122.25 & $8.58 \times 10^{29}$ & 8.81 & $5.16 \times 10^{33}$ & $4.75 \times 10^{34}$ \\
\hline 180609 & 5201816139806400896 & 18.57 & 3.00 & 0.20 & 2.49 & 0.41 & 294.79 & 94.21 & $8.97 \times 10^{29}$ & 0.89 & $2.36 \times 10^{32}$ & $2.17 \times 10^{33}$ \\
\hline 180609 & 5195663753775359232 & 20.66 & 3.39 & 0.19 & 2.86 & 1.56 & 146.53 & 111.87 & $1.74 \times 10^{29}$ & 4.09 & $1.04 \times 10^{32}$ & $9.60 \times 10^{32}$ \\
\hline 180607 & 5201720555311609344 & 18.5 & 2.70 & 0.15 & 2.08 & 0.5 & $2051.84+$ & 286.96 & $8.37 \times 10^{30}$ & 5.26 & $9.03 \times 10^{34}+$ & $8.30 \times 10^{35}$ \\
\hline 180607 & 5190564291860908672 & 16.2 & 2.37 & 0.11 & 1.74 & 0.07 & 384.36 & 164.45 & $2.29 \times 10^{31}$ & 0.16 & $1.43 \times 10^{33}$ & $1.32 \times 10^{34}$ \\
\hline
\end{tabular}


Table A1 - continued

\begin{tabular}{|c|c|c|c|c|c|c|c|c|c|c|c|c|}
\hline plitate & Gaia DR2 ID & $\begin{array}{l}\text { Quies- } \\
\text {-cent } \\
\text { mag } \\
(g)\end{array}$ & $\begin{array}{c}\text { Gaia } \\
\mathrm{BP}-\mathrm{RP}\end{array}$ & $\begin{array}{c}\text { WISE } \\
W 1-W 2\end{array}$ & $\begin{array}{c}\mathrm{SM} \mathrm{DR} 2 / 3 \\
\quad r-z\end{array}$ & $\begin{array}{c}\text { Flare } \\
\Delta \\
\text { mag } \\
(g)\end{array}$ & $\begin{array}{l}\text { Flare } \\
\text { Dura- } \\
\text {-tion }{ }^{a} \\
(\mathrm{~s})\end{array}$ & $\begin{array}{l}\text { Dist- } \\
\text {-ance } \\
(\mathrm{pc})\end{array}$ & $\begin{array}{c}\text { Quies- } \\
\text {-cent } \\
\text { flux } \\
\left(\text { erg s }^{-1}\right)\end{array}$ & $\begin{array}{l}\text { Rela- } \\
\text {-tive } \\
\text { flux } \\
\left(\sum\right)\end{array}$ & $\begin{array}{c}\text { Flare } \\
\text { energy }^{b} \\
\text { ( } g \text { band) } \\
\text { (erg) }\end{array}$ & $\begin{array}{c}\text { Flare } \\
\text { energy }^{b} \\
\text { (bol) } \\
(\mathrm{erg})\end{array}$ \\
\hline 160730 & 5190981075487146368 & 18.5 & 2.32 & 0.08 & 1.64 & 0.11 & 289.53 & 356.77 & $1.29 \times 10^{31}$ & 0.38 & $1.44 \times 10^{33}$ & $1.33 \times 10^{34}$ \\
\hline 160730 & 5191153870611593600 & 19.14 & 3.01 & 0.20 & 2.54 & 1.17 & 1161.24 & 159.25 & $1.43 \times 10^{30}$ & 7.43 & $1.23 \times 10^{34}$ & $1.13 \times 10^{35}$ \\
\hline 150114 & 5501797275898167936 & 19.78 & 3.17 & 0.23 & 2.67 & 0.83 & 432.12 & 203.98 & $1.30 \times 10^{30}$ & 3.34 & $1.88 \times 10^{33}$ & $1.73 \times 10^{34}$ \\
\hline 150114 & 5502892870516254720 & 19.04 & 2.90 & 0.20 & 2.32 & 0.24 & 580.09 & 185.39 & $2.12 \times 10^{30}$ & 1.64 & $2.02 \times 10^{33}$ & $1.86 \times 10^{34}$ \\
\hline 150114 & 5502128435056792448 & 18.62 & 2.67 & 0.15 & 2.11 & 0.11 & 190.8 & 282.69 & $7.27 \times 10^{30}$ & 0.27 & $3.85 \times 10^{32}$ & $3.54 \times 10^{33}$ \\
\hline 170205 & 5502472749699352320 & 17.17 & 2.82 & 0.17 & 2.28 & 0.14 & 243.50 & 75.21 & $2.08 \times 10^{30}$ & 0.31 & $1.56 \times 10^{32}$ & $1.44 \times 10^{33}$ \\
\hline 170205 & 5502028757456041600 & 16.46 & 2.70 & 0.10 & 2.14 & 0.17 & 440.71 & 119.97 & $9.58 \times 10^{30}$ & 0.32 & $1.35 \times 10^{33}$ & $1.24 \times 10^{34}$ \\
\hline 170206 & 5501843386667424640 & 20.16 & 3.39 & 0.23 & 2.99 & 1.07 & $242.77+$ & 246.33 & $1.34 \times 10^{30}$ & 3.77 & $1.22 \times 10^{33}+$ & $1.13 \times 10^{34}$ \\
\hline 170202 & 5502529344483998464 & 17.1 & 2.89 & 0.19 & 2.33 & 0.63 & 926.92 & 119.44 & $5.26 \times 10^{30}$ & 1.74 & $8.47 \times 10^{33}$ & $7.80 \times 10^{34}$ \\
\hline 150115 & 5502495805083748736 & 20.7 & 3.04 & 0.23 & 2.50 & 1.48 & 479.87 & 425.62 & $2.43 \times 10^{30}$ & 13.81 & $1.61 \times 10^{34}$ & $1.48 \times 10^{35}$ \\
\hline 150117 & 5502133893958218752 & 20.85 & 2.94 & 0.25 & 2.64 & 1.41 & 185.27 & 202.93 & $4.18 \times 10^{29}$ & 3.98 & $3.55 \times 10^{32}$ & $3.26 \times 10^{33}$ \\
\hline 160726 & 6438530639589294720 & 19.11 & 2.65 & 0.13 & 2.07 & 0.51 & 336.32 & 419.48 & $1.02 \times 10^{31}$ & 2.19 & $7.54 \times 10^{33}$ & $6.93 \times 10^{34}$ \\
\hline 160726 & 6435578657026534272 & 18.98 & 2.58 & 0.17 & 2.03 & 0.33 & 1059.51 & 343.08 & $7.69 \times 10^{30}$ & 3.24 & $2.64 \times 10^{34}$ & $2.43 \times 10^{35}$ \\
\hline 160729 & 6435523131689270656 & 19.08 & 2.82 & 0.18 & 2.29 & 0.26 & 145.21 & 241.59 & $3.48 \times 10^{30}$ & 0.51 & $2.59 \times 10^{32}$ & $2.38 \times 10^{33}$ \\
\hline 160729 & 6438148696738890880 & 17.19 & 2.74 & 0.18 & 2.19 & 0.07 & 385.34 & 107.44 & $3.92 \times 10^{30}$ & 0.26 & $3.94 \times 10^{32}$ & $3.64 \times 10^{33}$ \\
\hline 160802 & 6435368817806876416 & 18.96 & 2.05 & 0.15 & 1.90 & 0.33 & 386.79 & 411.33 & $1.13 \times 10^{31}$ & 1.00 & $4.39 \times 10^{33}$ & $4.04 \times 10^{34}$ \\
\hline 160802 & 6435441866611271296 & 20.14 & 2.89 & 0.17 & 2.39 & 1.73 & $288.99+$ & 277.84 & $1.73 \times 10^{30}$ & 12.11 & $6.06 \times 10^{33}+$ & $5.58 \times 10^{34}$ \\
\hline 160802 & 6438494836741682048 & 18 & 2.75 & 0.13 & 2.17 & 0.24 & 866.65 & 171.51 & $4.74 \times 10^{30}$ & 1.26 & $5.16 \times 10^{33}$ & $4.76 \times 10^{34}$ \\
\hline 160802 & 6438489128728588416 & 18.72 & 2.29 & 0.09 & 1.64 & 0.39 & 289.22 & 465.59 & $1.80 \times 10^{31}$ & 0.75 & $3.90 \times 10^{33}$ & $3.59 \times 10^{34}$ \\
\hline 160804 & 6435481586470460544 & 19.34 & 2.84 & 0.20 & 2.32 & 0.39 & 144.06 & 374.56 & $6.58 \times 10^{30}$ & 0.59 & $5.56 \times 10^{32}$ & $5.19 \times 10^{33}$ \\
\hline 160806 & 6438355434986673152 & 20.01 & 2.50 & 0.12 & 2.01 & 0.44 & 240.73 & 345.81 & $3.03 \times 10^{30}$ & 1.53 & $1.11 \times 10^{33}$ & $1.02 \times 10^{34}$ \\
\hline 160806 & 6435480903573366784 & 16.56 & 2.56 & 0.12 & 1.94 & 0.1 & 194.28 & 73.28 & $3.26 \times 10^{30}$ & 0.14 & $8.45 \times 10^{31}$ & $7.86 \times 10^{32}$ \\
\hline 160728 & 6438601004035363584 & 21.01 & 3.02 & 0.20 & 2.61 & 1.02 & 440.41 & 308.19 & $9.56 \times 10^{29}$ & 5.30 & $2.23 \times 10^{33}$ & $2.05 \times 10^{34}$ \\
\hline 160726 & 6400072128030483200 & 16.9 & 1.89 & -0.01 & 1.08 & 0.05 & 143.18 & 331.64 & $4.88 \times 10^{31}$ & 0.08 & $5.80 \times 10^{32}$ & $5.36 \times 10^{33}$ \\
\hline 160726 & 6400154934999897344 & 14.81 & 1.32 & -0.02 & 0.60 & 0.05 & 144.81 & 334.91 & $3.41 \times 10^{32}$ & 0.05 & $2.37 \times 10^{33}$ & $2.18 \times 10^{34}$ \\
\hline 160726 & 6400560066378703360 & 18.64 & 2.61 & 0.17 & 2.09 & 0.24 & 242.39 & 246.30 & $5.42 \times 10^{30}$ & 0.76 & $1.00 \times 10^{33}$ & $9.22 \times 10^{33}$ \\
\hline 150114 & 5481255306315173248 & 16.9 & 2.40 & 0.11 & 1.77 & 0.05 & 482.67 & 170.72 & $1.29 \times 10^{31}$ & 0.27 & $1.71 \times 10^{33}$ & $1.58 \times 10^{34}$ \\
\hline 150114 & 5482101964628144896 & 17.56 & 3.00 & 0.20 & 2.50 & 0.11 & 192.42 & 131.39 & $4.17 \times 10^{39}$ & 0.21 & $1.65 \times 10^{32}$ & $1.52 \times 10^{33}$ \\
\hline 150116 & 5482088598688453504 & 22 & 1.80 & - & - & 2.25 & 1921.56 & 155.11 & $9.73 \times 10^{28}$ & 20.13 & $3.77 \times 10^{33}$ & $3.46 \times 10^{34}$ \\
\hline 170202 & 4758254255601790592 & 18.71 & 2.53 & 0.08 & 1.94 & 0.63 & 664.45 & 353.73 & $1.05 \times 10^{31}$ & 2.32 & $1.62 \times 10^{34}$ & $1.49 \times 10^{35}$ \\
\hline 170207 & 5482097188624710400 & 14.24 & 1.19 & -0.06 & 0.43 & 0.16 & 146.64 & 306.72 & $4.48 \times 10^{32}$ & 0.17 & $1.20 \times 10^{34}$ & $1.10 \times 10^{35}$ \\
\hline 170202 & 4758720787833766400 & 19.89 & 2.38 & 0.10 & 1.67 & 0.19 & 237.89 & 427.90 & $5.17 \times 10^{30}$ & 0.40 & $4.94 \times 10^{32}$ & $4.54 \times 10^{33}$ \\
\hline 170205 & 5482097115608455296 & 18.26 & 2.28 & 0.12 & 1.61 & 0.08 & 145.82 & 484.05 & $2.97 \times 10^{31}$ & 0.14 & $6.12 \times 10^{32}$ & $5.63 \times 10^{33}$ \\
\hline 150116 & 4758312594141537152 & 20.65 & 3.33 & 0.24 & 2.71 & 1.08 & 1696.17 & 162.21 & $3.69 \times 10^{29}$ & 16.28 & $1.02 \times 10^{34}$ & $9.38 \times 10^{34}$ \\
\hline
\end{tabular}

Notes. ${ }^{a}+$ indicates that the flare fall was not fully within the time series data and this is a lower limit on the flare's duration.

${ }^{b}+$ indicates that the flare fall was not fully within the time series data and this is a lower limit on the flare energy released.

\section{APPENDIX B}

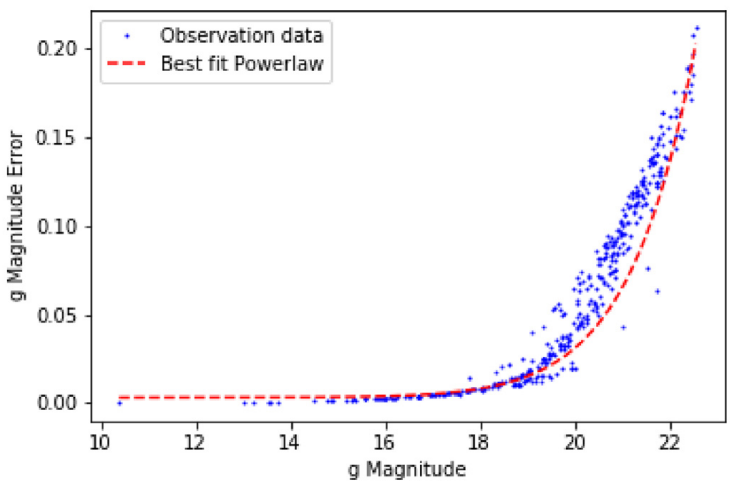

Figure B1. $g$ magnitude error versus $g$ magnitude for one central DECam CCD exposure of typical $20 \mathrm{~s}$ observations (e.g. airmass 1.5 , seeing $\sim 1.0$ arcsec FWHM).

This paper has been typeset from a $\mathrm{T}_{\mathrm{E}} \mathrm{X} / \mathrm{LT} \mathrm{E} \mathrm{X}$ file prepared by the author. 